Microglia under psychosocial stressors along the aging trajectory : Consequences on neuronal circuits, behavior, and brain diseases

Tian, Li

2017-10-03

Tian , L , Hui , C W , Bisht , K, Tan , Y, Sharma , K, Chen , S , Zhang , X \& Tremblay , M-E 2017 , ' Microglia under psychosocial stressors along the aging trajectory : Consequences on neuronal circuits, behavior, and brain diseases ' , Progress in

Neuro-Psychopharmacology \& Biological Psychiatry , vol. 79 , pp. 27-39 . https://doi.org/10.1016/j.pnpbp.2017.01.00

http://hdl.handle.net/10138/299386

https://doi.org/10.1016/j.pnpbp.2017.01.007

publishedVersion

Downloaded from Helda, University of Helsinki institutional repository.

This is an electronic reprint of the original article.

This reprint may differ from the original in pagination and typographic detail.

Please cite the original version. 


\title{
Microglia under psychosocial stressors along the aging trajectory: Consequences on neuronal circuits, behavior, and brain diseases
}

\author{
Li Tian a,b,*, Chin Wai Hui c ${ }^{\text {, }}$ Kanchan Bisht ${ }^{c}$, Yunlong Tan ${ }^{\mathrm{b}}$, Kaushik Sharma ${ }^{\mathrm{c}}$, Song Chen ${ }^{\text {b,d }}$, \\ Xiangyang Zhang ${ }^{\text {b,e }}$, Marie-Eve Tremblay ${ }^{\mathrm{c}, * *}$ \\ a Neuroscience Center, University of Helsinki, Viikinkaari 4, Helsinki FIN-00014, Finland \\ b Psychiatry Research Center, Beijing Huilongguan Hospital, Peking University, Beijing, China \\ c Axe Neurosciences, Centre de recherche du CHU de Québec, Québec, Canada \\ ${ }^{d}$ Beijing Key Laboratory of Mental Disorders and Center of Schizophrenia, Beijing Institute for Brain Disorders, Beijing Anding Hospital, Capital Medical University, China \\ e Department of Psychiatry and Behavioral Sciences, The University of Texas Health Science Center at Houston, Houston, TX, USA
}

\section{A R T I C L E I N F O}

\section{Article history:}

Received 11 November 2016

Received in revised form 30 December 2016

Accepted 13 January 2017

Available online 15 January 2017

\section{Keywords:}

Microglia-neuron interactions

Inflammation

Oxidative stress

Psychosocial stress

Aging

\begin{abstract}
A B S T R A C T
Mounting evidence indicates the importance of microglia for proper brain development and function, as well as in complex stress-related neuropsychiatric disorders and cognitive decline along the aging trajectory. Considering that microglia are resident immune cells of the brain, a homeostatic maintenance of their effector functions that impact neuronal circuitry, such as phagocytosis and secretion of inflammatory factors, is critical to prevent the onset and progression of these pathological conditions. However, the molecular mechanisms by which microglial functions can be properly regulated under healthy and pathological conditions are still largely unknown. We aim to summarize recent progress regarding the effects of psychosocial stress and oxidative stress on microglial phenotypes, leading to neuroinflammation and impaired microglia-synapse interactions, notably through our own studies of inbred mouse strains, and most importantly, to discuss about promising therapeutic strategies that take advantage of microglial functions to tackle such brain disorders in the context of adult psychosocial stress or aging-induced oxidative stress.
\end{abstract}

(c) 2017 Elsevier Inc. All rights reserved.

\section{Introduction}

The reciprocal relationships between the nervous and immune systems have been intensively investigated in recent years, attracting more and more research interest, especially regarding the brain resident innate immune cells - microglia (Prinz and Priller, 2014; Ueno and Yamashita, 2014; Yirmiya et al., 2015). Microglia and the immune genes they express are now being recognized as key mediators of neuronal circuitry formation, function, and plasticity during normal physiological conditions (Hong et al., 2016; Salter and Beggs, 2014; Tay et al., 2016). Abnormality of these crucial microglial processes contributes to neurodevelopmental defects resulting in several mental disorders such as autism, schizophrenia, and depression (Laskaris et al., 2016; Yirmiya et al., 2015).

Microglia are derived from yolk-sac myeloid progenitors invading the brain during embryonic development (Ginhoux et al., 2010;

\footnotetext{
* Correspondence to: L. Tian, Neuroscience Center, University of Helsinki, Viikinkaari 4, Helsinki FIN-00014, Finland.

** Corresponding author.

E-mail addresses: li.tian@helsinki.fi (L. Tian), marie-eve.tremblay@crchudequebec.ulaval.ca (M.-E. Tremblay).
}

Kierdorf et al., 2013). They are the only immune cells that permanently reside in the brain, alongside neurons, astrocytes, and oligodendrocytes. Microglial interactions with synapses were first described during development, and subsequently across the lifespan (Bisht et al., 2016; Paolicelli et al., 2011; Schafer et al., 2012; Tremblay et al., 2010, 2012; Wake et al., 2009). Microglia-synapse interactions are crucial for remodeling of neuronal circuits in the mature brain, and involve several mechanisms of which the best characterized are: 1 ) synaptic stripping, i.e. the physical separation of pre- and post-synaptic elements by intervening microglial processes, and 2) the phagocytic elimination or 'pruning' of axon terminals and dendritic spines (reviewed in Kettenmann et al., 2013). Recently, variations of the complement component (C) 4 resulting in increased brain levels of $\mathrm{C} 3$, a known mediator of microglial synaptic pruning, were strongly linked to the susceptibility to schizophrenia in humans (Sekar et al., 2016). In addition, microglia release a number of neurotrophic factors such as glia-derived growth factor, brain-derived neurotrophic factor (BDNF), and insulin growth factor- 1 (IGF-1) that are essential for neurogenesis, as well as neuronal circuit maturation and function (Parkhurst et al., 2013; Sierra et al., 2014; Ueno et al., 2013; Ziv and Schwartz, 2008).

Psychosocial stress is a state of mental or emotional strain or tension that results from adverse or demanding circumstances. It has 
multifaceted causes and occurs frequently over the course of a lifetime with varying dimensions and intensity, irrespective of a person's occupation or position within a society (Machado et al., 2014). Persistent maladaptive response to stress is associated with anxiety, depression, and several other neuropsychiatric manifestations (McEwen et al., 2015; Prenderville et al., 2015). The relationship between psychosocial stress and microglial phenotypic transformation, defined in terms of increased density, change of morphological indices, and exaggerated pro-inflammatory gene expression, is documented across animal and human studies. Microglial transformation has been identified in the hippocampus, prefrontal cortex (PFC), amygdala, and paraventricular nucleus of the hypothalamus, among other brain regions associated with emotion and the development of mental disorders. For instance, adult rats previously exposed to prenatal psychosocial stress display an increased density of ionized calcium-binding adaptor molecule 1 (Iba1)-immunopositive $(+)$ microglia, enhanced levels of proinflammatory cytokines and reactive nitrogen species, as well as lowered levels of neurotrophic factors in the hippocampus and PFC (Slusarczyk et al., 2015). Chronic exposure to psychosocial stress may, therefore, utilize this arm to trigger and/or exacerbate mental disorders (Calcia et al., 2016; Lehmann et al., 2016; McKim et al., 2016; Ramirez et al., 2015).

When psychosocial stress becomes a chronic condition, people are more likely to develop and experience a more rapid progression of aging and various age-related diseases (Fidler et al., 2011; Hou et al., 2014; Mo et al., 2014). Aging can 'prime' microglial transformation - defined as a phenotypic imprinting that occurs upon exposure to stressful challenges of physiological or psychological nature - and exacerbate neuroinflammation (Cornejo and von Bernhardi, 2016; Niraula et al., 2017). Psychosocial stress and aging similarly potentiate cellular aging, oxidative stress, and neuroinflammation (Prenderville et al., 2015), and may thereby jointly accelerate cognitive aging and neurodegenerative diseases (Miller and Sadeh, 2014). Anxiety and depression are being increasingly recognized as risk factors for the development of neurodegenerative diseases that comprise the highly-prevalent Alzheimer's disease (AD) (Alkadhi, 2012; Norton et al., 2014), and preclinical studies demonstrate that chronic stress decreases the levels of memory-related signaling molecules in the hippocampus and exacerbates cognitive impairment in AD animal models (Alkadhi and Tran, 2015; Srivareerat et al., 2009).

Psychosocial stress triggers activation of the hypothalamicpituitary-adrenal (HPA) axis leading to the release of glucocorticoid hormones from the adrenal glands, and prolonged exposure to corticosterone, which is the main glucocorticoid in rodents, was shown to exert detrimental changes in the brain inducing atrophy of neuronal dendrites (Sapolsky et al., 1985), synaptic loss (Liston and Gan, 2011; Tata et al., 2006), and eventually $N$-methyl-D-aspartate (NMDA) receptor-mediated excitotoxic death of neurons (Takahashi et al., 2002), caused by an excessive release of glutamate (SteinBehrens et al., 1994). Synaptic loss is considered the best pathological correlate of cognitive decline across a myriad of brain disorders that include stress-induced major depression, schizophrenia, and neurodegenerative diseases such as AD (Duman and Aghajanian, 2012; Jurgens et al., 2016; McEwen et al., 2015; Spires-Jones and Hyman, 2014).

In this review, we explore the relevance of targeting microgliasynapse interactions to spare cognitive functions, which can be compromised along the aging trajectory by psychosocial stress. We hereby present the characteristics of microglia under psychosocial stressors, their implication in mental disorders occurring on the aging trajectory, through phenotypic alterations, remodeling of neuronal circuits (via synaptic formation, pruning, and neurogenesis), and mediation of oxidative stress and neuroinflammation. Our own studies using inbred strains of mice are discussed. We propose therapeutic avenues to promote stress resilience of microglia for a healthy cognitive aging.

\section{Microglial transformation upon psychosocial stress across the lifespan}

Chronic psychosocial stress is an environmental risk factor that exerts a profound influence on microglia and the brain's inflammatory milieu. Over the past ten years, various stress models induced by maternal separation, social isolation, chronic restraint, repeated social defeat, forced swim, foot shock and an unpredictable series of varying stressors, etc., have been used to study how stress affects microglia and thereby leads to exacerbated behavioral deficits in rodents (Calcia et al., 2016; Lehmann et al., 2016; McKim et al., 2016; Ramirez et al., 2015).

The impact of stress on microglia already occurs at early stages of brain development, especially when the exposure takes place during critical time windows of vulnerability (Bilbo and Schwarz, 2012; Castanon et al., 2015). Maternal stress profoundly affects the physiological and behavioral characteristics of the offspring, and has been linked to symptoms of depression and anxiety later in life (Knuesel et al., 2014). However, the responsible mechanisms remain largely undetermined. In a recent study, a 72-hour sleep deprivation occurring during the last gestational trimester in rats was shown to significantly increase Iba ${ }^{+}$microglial density in the hippocampus of pre-puberty male offspring, alongside a decline in neurogenesis and an impairment of hippocampus-dependent spatial learning and memory (Zhao et al., 2015). These alterations could be reversed by pre-treatment with minocycline, a broad spectrum tetracyclinederived antibiotic commonly used to prevent microglial transformation, suggesting a role for microglia in mediating maternal sleep deprivation-induced cognitive deficits (Zhao et al., 2015). Similarly, prenatal stress induced by exposure to bright light for $45 \mathrm{~min}$ in mice was shown to adversely exacerbate microglia-mediated inflammatory response in the hippocampus of adult female offspring (Diz-Chaves et al., 2012). The hippocampus expresses a higher level of glucocorticoid receptor (GR) as compared to other brain regions (Reul and de Kloet, 1985), and since GR is the main receptor for the stress hormone corticosterone, the hippocampus is highly vulnerable to the harmful effects of psychosocial stress (McEwen et al., 1992).

Following lipopolysaccharide (LPS) injection, prenatally stressed adult mice compared with non-stressed mice showed an increased density of microglia with amoeboid cell bodies and numerous shorter and thicker processes in the dentate gyrus (DG) (Diz-Chaves et al., 2012). Prenatal stress, induced through 20 min of maternally forced swim on embryonic days 10-20, also increased microglial density in newborn rats across several brain areas that include the frontal, entorhinal and parietal cortices, as well as the subcortical basal ganglia, thalamus, medulla, septum, corpus callosum and internal capsule (Gomez-Gonzalez and Escobar, 2010). At postnatal day 10, this increased microglial density returned to baseline level, similar to non-stressed control rats, among all brain areas, with the exception of the corpus callosum where a reduction of ramified microglia was retained in the prenatal stress group (Gomez-Gonzalez and Escobar, 2010). Similarly, neonatal maternal separation led to alterations of microglial morphology in the somatosensory cortex, which persisted into adulthood, suggesting that microglial priming results in their exaggerated response to secondary stressful stimuli occurring later in life (Takatsuru et al., 2015). Microglial priming by stressful events during prenatal periods was proposed to explain their involvement in a number of affective disorders and schizophrenia (Hanamsagar and Bilbo, 2016).

In adulthood, a number of studies using various murine stress models revealed that exposure to acute and chronic psychosocial stressors at maturity can prime microglia at both morphological and molecular levels, as will be elaborated below. Several other recent reviews covering related topics are also available for readers (Calcia et al., 2016; Delpech et al., 2015a; Pearson-Leary et al., 2015; Reader et al., 2015; Walker et al., 2014). 


\subsection{Changes of density and morphology}

The effects of stress on microglia can be multiple, depending on the type, duration, and frequency of the stressor-exposure. Nevertheless, many studies using various rodent models and stress paradigms consistently demonstrated that psychosocial stress can alter microglial cell density and morphology, using the OX-42 and Iba1 markers, which do not only label microglia, but also the bone marrow-derived monocytes and macrophages found in the brain under certain conditions. Considering that resident microglia represent the majority of brain myeloid cells, for simplicity we will solely refer to microglia in the subsequent paragraphs. For more information regarding the respective contributions of microglia $v s$ bone marrow-derived monocytes to the stress response, our readers are referred to a recent review by Wohleb and Delpech (2017).

In particular, chronic unpredictable stress (CUS) induced by restraint or forced swim in rats and mice has been shown to significantly increase microglial density among the hypothalamus, thalamus, and hippocampus, as assessed by immunostaining for OX-42, which labels both CD11b and CD11c (Sugama et al., 2007). CD11b composes the complement receptor 3 that is expressed by microglia and is involved in synaptic pruning (Schafer et al., 2012), while CD11c is considered a marker of dendritic cells throughout the brain and periphery (D'Agostino et al., 2012). Similarly, an increased number of Iba $1^{+}$microglia was observed in the prelimbic and infralimbic cortices following chronic restraint stress (30-minute daily exposure for 14 days) (Kopp et al., 2013). After chronic restraint stress in rats, Tynan et al. also observed a significant increase in Iba $1^{+}$microglial density among a number of brain regions implicated in the stress response, including the anterior cingulate cortex (ACC), infralimbic and prelimbic medial PFC, nucleus accumbens, medial amygdala, bed nucleus of the stria terminalis, CA3 region of the hippocampus, and periaqueductal grey (Tynan et al., 2010). A significant increase in Iba + microglial density was additionally observed in response to another stress paradigm - acute social defeat stress, in which the experimental mice were repeatedly stressed for 3 days. These changes were encountered within different stress-responsive brain regions, namely the infralimbic, prelimbic and ACC cortices, the basolateral amygdala, DG, nucleus accumbens, and the piriform cortex (Lehmann et al., 2016).

Kreisel et al. further revealed that microglial density varies over the course of stress response. The initial increase of $\mathrm{Iba}^{+}{ }^{+}$microglial density induced by 2 days of CUS in mice was followed, 2 days later, by a return to baseline density within the DG (Kreisel et al., 2014). In this case, the appearance of caspase $-3^{+}$microglia 3 days after CUS also suggested that a subset of stressed microglia would undergo apoptosis (Kreisel et al., 2014). An increased proportion of microglia displaying morphological alterations was found to accompany these changes in density. For instance, alongside an increased density, a significant although partial decrease of $\mathrm{OX}-42^{+}$microglia showing an increased surface area and number of thick and short processes was described in the periaqueductal grey, a region modulating pain, upon restraint or forced swim stress in rats and mice (Sugama et al., 2007). These changes were not observed in mice deficient for the pro-inflammatory cytokine interleukin (IL)-18, indicating that microglial response to stress is partially mediated by IL-18, at least under this paradigm (Sugama et al., 2007).

In other rodent studies, chronic stress was similarly shown to alter microglial morphology in a subset of stress-responsive brain regions, inducing either hyper-ramification - as characterized by an increase in the total number of branching points and an elongation of processes (Hinwood et al., 2012, 2013) - or dystrophic morphologies - defined by a retraction of processes and a decrease in their branching points or arborization area (Kreisel et al., 2014; Milior et al., 2015; Wohleb et al., 2014). Microglial hyper-ramification was recently described in the mouse DG following 5 days of repeated forced swim stress (Hellwig et al., 2016). On the opposite, repeated social defeat was found to de-ramify microglia in the mouse amygdala and hippocampus within 8 days, and in the PFC after 24 days of stress (Wohleb et al.,
2014). CUS, induced either conventionally or by an Intellicage system designed for behavioral monitoring with minimal human intervention, similarly reduced microglial process length and arborization area, in the hippocampus CA1 or DG after 2 or 5 weeks of exposure (Kreisel et al., 2014; Milior et al., 2015). For both hyper-ramified and dystrophic phenotypes, an enlargement of microglial cell body was additionally noted (Hinwood et al., 2012, 2013; Kreisel et al., 2014), suggesting an increased transcriptional activity (Dungrawala et al., 2010), which might be related to microglial release of either anti- or proinflammatory mediators, as well as trophic factors. Kreisel et al. additionally revealed that the initial increase in microglial cell body area observed after 2 days of CUS in mice returned to baseline when measured another 2 days later, within the DG (Kreisel et al., 2014).

Overall, these changes of microglial density indicate a greater need for microglial interventions within the stressed brain - taking place most likely at neuronal circuits - through oxidative stress, neuroinflammation and/or synaptic remodeling, as will be discussed below. Microglial morphological changes also suggest a continuum that may represent their functional repertoire recruited along the process of stress adaptation. Nevertheless, how the phenomena of hyper-ramification vs deramification may differently modify - structurally and functionally microglial interactions with newborn neurons, synapses and other neuronal elements in the brain parenchyma remains to be determined.

\subsection{Changes of inflammatory phenotype and association with anxiety}

Researchers have also applied various approaches to characterize microglial changes induced by stress exposure at the molecular level. Such knowledge will undoubtedly provide deeper insights into the pathogenesis and pathophysiology of mental disorders. In animal behavioral researches, in addition to comparing inter-individual responses to stress, inbred mice were utilized to model the inter-individual variability in behavioral traits that include, but are not limited to, anxiety and psychosocial stress responses (Hovatta and Barlow, 2008; Wahlsten, 2012). A number of research studies have used inbred strains to discover novel candidate genes related to psychiatric traits such as schizophrenia and anxiety (Mozhui et al., 2010; Stevens et al., 1996; Yang et al., 2008). A previous study showed that lipopolysaccharidebinding protein (LBP), a protein involved in the acute-phase immunologic response to gram-negative bacteria, is dramatically decreased in the hippocampus of BALB/cByj mice, an inbred strain with high anxiety-trait, when the pups were exposed to maternal separationinduced early life stress. These LBP-deficient mice additionally showed increased anxiety-like behaviors (Wei et al., 2012).

The characterization of microglial molecular 'signature' has utilized polarizing phenotypes inspired by previous investigation on macrophages. Macrophages were divided into the classic, pro-inflammatory (M1) phenotype - defined as an increased release of pro-inflammatory cytokines such as tumor necrosis factor alpha (TNF- $\alpha$ ), IL-1 $\beta$, IL-6, IL-12 and IL-18, as well as a reduction in microglial phagocytosis - vs the alternative, anti-inflammatory (M2) phenotype - defined as an increased production of anti-inflammatory IL-4, IL-10, IL-13 and transforming growth factor-beta (TGF- $\beta$ ), along with an increase in phagocytic ability. These M1 vs M2 phenotypes should, however, be considered as representing the opposing ends of a wide and mutually non-exclusive spectrum of myeloid phenotypic states (Sica and Mantovani, 2012), especially in vivo, a view that is reaching consensus both in the fields of microglia and macrophages (Murray et al., 2014; Ransohoff, 2016a).

Increasing evidence suggests that microglial transformation is finely tuned to the physiological and pathophysiological needs of the central nervous system (CNS), in which these cells adopt various intermediate phenotypes depending on the nature of the activating stimuli (Franco and Fernandez-Suarez, 2015). As a consequence, the M1 vs M2 categorization may under-represent the versatility of microglial functions. Even though the distinctive features of M1 and M2 microglia still need 
to be better defined in vivo, cumulative evidence indicates that microglia can travel the alternative activation pathway, express M2-type markers, and promote neuroprotection under particular contexts (Franco and Fernandez-Suarez, 2015). Whether psychosocial stress may promote microglial polarization towards one $v s$ another extreme remains to be determined, but accumulating evidence has emerged. For instance, enriched environment in a mouse model of depression induced by chronic corticosterone administration was shown to block pro-inflammatory cytokine gene induction and promote arginase 1 (Arg1) mRNA expression in sorted microglia, indicating an M2a-type of transition, and the effect was abolished in adiponectin-knock-out (KO) microglia (Chabry et al., 2015).

We additionally used inbred mice to study the relationship between microglial transformation and anxiety levels, which revealed that strains showing higher anxiety, such as DBA/2J and 129S2/Sv mice, had higher ratios of the pro-inflammatory major histocompatibility complex (MHC) class $\mathrm{II}^{+}$M1-like $v s$ the anti-inflammatory CD206 ${ }^{+}$M2-like microglia, compared with strains with lower anxiety, such as $\mathrm{C} 57 \mathrm{Bl} / 6 \mathrm{~J}$ and FVB/NJ mice. These differences between inbred strains were found to be exacerbated after a peripheral LPS challenge known to prime the M1 polarization of macrophages and microglia (Orihuela et al., 2016). Furthermore, we found that the pro-inflammatory cytokines $I l 1 \mathrm{~b}$, Il6, and Tnf, and ratio of the M1/M2 marker genes, Nos2/Arg1, were elevated in the high anxiety strains undergoing LPS challenge (Li et al., 2014).

By further comparing transcriptomic microarray data of eight inbred strains, we identified a cluster of 23 brain immune genes that are constitutively expressed at different levels among these strains, most of which were innate immune genes that have been either demonstrated or speculated to be associated with microglial functions (Ma et al., 2015a, $2015 b)$. Out of these inbred mouse strains, we and other researchers have found that C57BL/ 6 and DBA/2 mice, which are the most extensively studied ones, differ in many basal behavioral properties including increased anxiety (Hovatta et al., 2005; Kulesskaya et al., 2014; Mozhui et al., 2010; Voikar et al., 2005) and reduced sociability (Ma et al., 2015b; Moy et al., 2007). We also found that C57BL/6 and DBA/2 mice responded differently to psychosocial stress in changing their social and cognitive behaviors (Kulesskaya et al., 2014). Notably, our work revealed that these two strains differed in their expression of several genes shown to regulate synaptic pruning or plasticity, such as $C 1 q b$, Cx3cl1, H2-d1, and H2-k1 (Ma et al., 2015a, 2015b). C1qb is an important subcomponent of the classical complement system demonstrated to mediate microglial synaptic pruning in development, aging, and $\mathrm{AD}$ (Hong et al., 2016). Cx3cl1 encodes the fractalkine ( $\mathrm{CX}_{3} \mathrm{CL} 1$ ), a ligand that, along with its receptor $\mathrm{CX}_{3} \mathrm{CR} 1$ expressed on microglia, is essential for healthy neuron-microglial crosstalk (Arnoux and Audinat, 2015; Paolicelli et al., 2014). H2-d1 and H2-k1, which encode the MHC class I antigen D-B-alpha-chain and antigen A, respectively, are important for the presentation of foreign antigens to the immune system (Neefjes et al., 2011), and also for synaptic elimination, neuronal wiring, and long-term synaptic plasticity during brain development (Adelson et al., 2016; Lee et al., 2014).

Besides ours, a few other previous studies have identified that psychosocial stress can induce changes in microglial gene expression, with different outcomes depending on the stress paradigm. Although some studies using chronic restraint stress on rats did not find any evidence of increased IL-1 $\beta$, MHC class II and CD68 (a marker of lysosomal activity) in rat microglia (Hinwood et al., 2013; Tynan et al., 2010), acute stress induced by tail- or foot-shock in rats was shown to upregulate microglial MHC class II, Il1 b or Cd14, a monocyte marker, and to downregulate Cd200r (Blandino et al., 2009; Frank et al., 2007). Cd200r, encoding a receptor regulating myeloid functions, was found to exert a calming influence on microglial phenotype upon binding to its ligand CD200 (Hanisch and Kettenmann, 2007; Jenmalm et al., 2006). In addition, the activity of P2X7R purinergic receptor expressed by microglia was shown to result in proliferation and transformation of rat primary hippocampal microglia even in the absence of pathological insult (Monif et al.,
2009). By using a P2X7R antagonist or KO mice of the inflammasome component Nlrp3, Iwata et al. recently demonstrated that the release of IL-1 $\beta$ and TNF- $\alpha$ normally induced by acute restraint stress can be completely blocked in the hippocampus (Iwata et al., 2016). Moreover, administration of the P2X7R antagonist reversed anhedonic and anxiety-like behaviors caused by CUS (Iwata et al., 2016). Conversely, an inflammation restricted to the insular cortex, as induced by LPS infusion, was shown to enhance associative taste memory through a purinergic modulation of glutamatergic AMPA (but not NMDA) receptors (Delpech et al., 2015b). In another paradigm of CUS, depression-susceptible C57BL/6J mice, when compared to their resilient counterparts, showed elevated expression of TNF in the PFC and indoleamine-2,3-dioxygenase (IDO) in the raphe, accompanied by an increased density of Iba $1^{+}$microglia in the PFC (Couch et al., 2013). In addition, various stress paradigms can potentiate microglia to produce more pro-inflammatory cytokines upon stimulation with LPS (de Pablos et al., 2014; Frank et al., 2007; Johnson et al., 2013; Wohleb et al., 2012), while a combination of innate immune challenge and psychosocial stressors was shown to summate into exaggerated anxiety and social deficits in rodents (Bilbo and Schwarz, 2012; Giovanoli et al., 2013; Wohleb et al., 2012).

Recently, repeated social defeat in mice was revealed to increase expression of a selection of microglial, or strictly saying, CD11 ${ }^{+}$-cell-specific genes in the brain. Notably in these studies, the pro-inflammatory cytokines Ill $\beta$ and $\operatorname{Tnf} \alpha$ were found to be upregulated at early stages (within $12 \mathrm{~h}$ ) of stress, contrary to Il6, which was only upregulated after 8 days of stress, and to $C d 14$ and $C \times 3 c r 1$, which were elevated throughout the 24 days of stress (McKim et al., 2015; Wohleb et al., 2011, 2014). The above-described effects have been accredited to an activation of the sympathetic nervous system, as blocking its activity by subcutaneous injection of propranolol (a $\beta$ blocker) or guanethidine (a norepinephrine release inhibitor) counteracted the stress-induced upregulation of pro-inflammatory cytokines in the brain (Blandino et al., 2009; McKim et al., 2015), whereas intracerebroventricular administration of the $\beta$-adrenergic agonist isoproterenol amplified it (Johnson et al., 2013). Intriguingly, such effect was found to be opposite to the anti-inflammatory effect exerted by epinephrine and norepinephrine on immune cells in the periphery, since the expressions of both early and late genes induced for instance by LPS, including $T n f$ and Il6, were dampened in rodents (Bellinger and Lorton, 2014; Martelli et al., 2014). Whether such discrepancy is due to the differential expression and activity of adrenergic receptors or to some other confounding factors in microglia vs splenic macrophages is currently unclear.

The above data reporting changes in microglial density, morphology, and gene expression upon psychosocial stress have motivated the development of microglial modulators to tackle various brain diseases including mental disorders (Biber et al., 2016). Under stress paradigms, minocycline was demonstrated to reverse $I l 1 \mathrm{~b}$ upregulation (Blandino et al., 2009), induce expression of M2 microglial markers, which include Arg1 - an enzyme that contributes to wound healing, Ym1 (Chi3l3) - a heparin-binding lectin, FIZZ1 - a cysteine-rich protein that promotes extracellular matrix deposition, and CD206 - a scavenging mannose receptor (Burke et al., 2014; Zhao et al., 2015). It also prevented microglial morphological transformation (Hinwood et al., 2012; Kreisel et al., 2014), and rescued cognitive deficits and depression-like behaviors, of stressed rodents (Burke et al., 2014; Hinwood et al., 2013; Kreisel et al., 2014). However, in these studies, minocycline exerted beneficial effects only when it was administered in prophylaxis prior to stress induction, showing minimal therapeutic outcomes after the onset of stress-related symptoms (Kreisel et al., 2014).

\section{Microglial implication in mental disorders across the lifespan}

\subsection{Evidence from human studies}

Given the importance of microglia and their mediators for normal brain homeostasis, it is not surprising that disturbances in these cells 
are encountered across various mental disorders, such as schizophrenia and depression. Primary and secondary 'microgliopathologies' are considered to be implicated in numerous diseases and have been extensively studied in recent years (Biber et al., 2016; Prinz and Priller, 2014; Yirmiya et al., 2015). In humans, changes in microglial phenotypic transformation under psychiatric disturbance were observed in postmortem brain samples (Steiner et al., 2008; Torres-Platas et al., 2014). For example, microglial cells showing increased staining for Iba1 and de-ramified morphologies were encountered in the white matter of the dorsal PFC and dorsal ACC of suicide-committers (Schnieder et al., 2014; Torres-Platas et al., 2014). In both regions, there was also an increased density of $\mathrm{CD} 45^{+}$myeloid cells around the blood vessels (Schnieder et al., 2014; Torres-Platas et al., 2014). In addition, increased 'microgliosis' or density of MHC class $\mathrm{II}^{+}$cells was reported in the dorsolateral PFC, ACC, and mediodorsal thalamus in suicidecommitters, irrespective of their schizophrenia or depression diagnosis (Steiner et al., 2008).

In parallel, clinical researchers have been employing the latest imaging technologies to visualize microglial activity in vivo and to correlate changes in their expression of phenotypic markers with cases of depression and other psychiatric disorders. For example, positron emission tomography (PET) imaging has been conducted by using ${ }^{11} \mathrm{C}-\mathrm{PK} 11195-\mathrm{a}$ ligand for the neuroinflammation marker translocator protein $18 \mathrm{kDa}$ (TSPO) - to find a microglial association with depression (Setiawan et al., 2015). Compared to ${ }^{11} \mathrm{C}$-PK11195, the second-generation TSPO radiotracers enable wider use in different research facilities and improve the signal-to-noise ratio (Vivash and O'Brien, 2016). Expressed on the outer mitochondrial membrane, TSPO, which is mainly but not exclusively expressed by microglia, is essential for steroid hormone synthesis by allowing the flow of cholesterol into mitochondria and is therefore involved in mitochondrial functioning, and shows lower expression in the healthy brain as compared to neuropathological conditions (Rupprecht et al., 2010). Overall, increased microglial density and morphological phenotypic transformation (such as enlarged cell bodies and de-ramified processes) and increased TSPO expression were widely documented in post-mortem brain specimens across autism (Morgan et al., 2010), schizophrenia (Bloomfield et al., 2016; Steiner et al., 2008; van Berckel et al., 2008), depression (Steiner et al., 2008) and bipolar disorder (Haarman et al., 2014) patients.

\subsection{Microglial remodeling of neuronal circuits}

In the absence of pathological insult, microglia continuously scan the brain parenchyma with their profusion of highly ramified, motile processes. This extreme dynamism has been discovered using non-invasive transcranial two-photon in vivo imaging of transgenic mice, in which microglia are fluorescently labeled (reviewed in Tay et al., 2016). Subsequently, ultrastructural analyses revealed that $94 \%$ of all microglial processes directly contact synaptic elements in the healthy brain (Tremblay et al., 2010). Axon terminals, dendritic spines, perisynaptic astrocytic processes, and synaptic clefts were found to be contacted, in decreasing order of frequency, in the adolescent mouse cerebral cortex (Tremblay et al., 2010). These synaptic interactions are far from being random but rather continuously modulated by synaptic plasticity, as well as by sensory and behavioral experiences (Milior et al., 2015; Pfeiffer et al., 2016; Tremblay et al., 2010, 2012). Overall, these findings led to the proposition that microglial 'immune' surveillance targets mainly synaptic elements under normal physiological conditions. In terms of molecular underpinnings, purinergic signaling - through the microglial P2RY12 (Haynes et al., 2006) - was shown to drive microglial response to a laser-induced injury in the mouse cerebral cortex in vivo (Davalos et al., 2005). Recently, microglial dynamics were also found to be controlled by the TAM receptor tyrosine kinases MerTK and Axl, known to regulate the innate immune responses of dendritic cells and macrophages and the engulfment of apoptotic cells by phagocytes (Lemke, 2013; Zagorska et al., 2014). Precisely, microglia from TAM-deficient mice displayed reduced process motility and delayed convergence towards sites of laser-induced injury in the cerebral cortex in vivo (Fourgeaud et al., 2016). However, how purinergic signaling could interact with the TAM receptor signaling pathways still remains elusive.

Surveilling microglia actively maintain health - a homeostatic process that strongly relies on their involvement with the guidance of axonal projections, the maturation, function and plasticity of synapses, and the integration of newborn neurons into the hippocampal and olfactory bulb circuitries (Cunningham et al., 2013; Hong et al., 2016; Salter and Beggs, 2014; Sierra et al., 2013; Squarzoni et al., 2014; Tay et al., 2016). Pre-synaptic axonal terminals, post-synaptic dendritic spines, and even entire synapses are engulfed by microglia in the healthy brain, as revealed using electron microscopy and/or super-resolution imaging within different regions (Paolicelli et al., 2011; Schafer et al., 2012; Tremblay et al., 2010). In the context of adult neurogenesis, surveilling microglia eliminate - by means of phagocytosis - the excess of newborn neurons dying by apoptosis in both neurogenic niches (Fourgeaud et al., 2016; Ribeiro Xavier et al., 2015; Sierra et al., 2010). For more information, our readers are referred to recent specialized reviews on the microglial regulation of neurogenesis, synaptic maturation, function and plasticity (Hong and Stevens, 2016; Valero et al., 2016).

In terms of underlying molecular mechanisms, $\mathrm{CX}_{3} \mathrm{CL} 1-\mathrm{CX}_{3} \mathrm{CR} 1$ signaling - which is the best-characterized axis of neuron-microglia interaction (Ransohoff and El Khoury, 2015) - was shown to mediate all the above-described physiological roles of microglia (reviewed in Arnoux and Audinat, 2015; Paolicelli et al., 2014) that are also profoundly affected by psychosocial stress (Knuesel et al., 2014; Lemaire et al., 2000; Takeuchi and Kawashima, 2016). Indeed, microglial $C_{3}$ CR1 is now considered to be crucially involved in developmental and adult neurogenesis, axon guidance, as well as synapse formation, maturation, function, and plasticity under normal physiological conditions, as notably demonstrated by the various defects described in $\mathrm{CX}_{3} \mathrm{CR} 1-\mathrm{KO}$ mice (Hoshiko et al., 2012; Maggi et al., 2009, 2011; Paolicelli et al., 2011; Rogers et al., 2011; among others). In addition, studies in mice showed that microglial release of BDNF promotes the formation of dendritic spines in the cerebral cortex during motor learning on a rotarod (Parkhurst et al., 2013), the classical complement cascade induces microglial pruning of axon terminals and dendritic spines across several brain regions during development, schizophrenia, aging, and in AD (Hong et al., 2016; Sekar et al., 2016; Shi et al., 2015; Stevens et al., 2007), and purinergic signaling through microglial P2RY12 mediates experience-dependent plasticity in the visual system (Sipe et al., 2016).

Under pathological states that include psychosocial stress response in mice, our work recently uncovered the existence of an ultrastructurally distinct microglial phenotype, especially with respect to synaptic interactions (Bisht et al., 2016). These cells appeared to be extremely active, frequently reaching for synaptic clefts, while extensively encircling axon terminals, dendritic spines and entire synapses with their highly ramified and thin processes. They strongly express CD11b, which is involved in microglia-mediated synaptic pruning (Schafer et al., 2012), specifically in their processes encircling synaptic elements. These cells also express myeloid-cell-specific triggering receptor expressed on myeloid cells (TREM2), when associated with amyloid- $\beta$ plaques in an APP-PS1 mouse model (Bisht et al., 2016). In AD pathology, TREM2 ${ }^{+}$cells were recently shown to express the phagocytic effectors MerTK and Axl (Savage et al., 2015). These cells were rare under steady state conditions, but became prevalent upon CUS and repeated social defeat, as well as in $\mathrm{CX}_{3} \mathrm{CR} 1$-deficiency, aging and $\mathrm{AD}$ pathology, and account for almost one-third of the microglial population under stress (Bisht et al., 2016). They exhibit several signs of oxidative stress including a condensed (electron-dense) cytoplasm and nucleoplasm, giving them a 'dark' appearance in electron microscopy, accompanied by endoplasmic reticulum dilation - the best characterized sign of oxidative stress at the ultrastructural level, mitochondrial disruption and heterochromatin-remodeling associated with epigenetic alteration (Bisht et al., 2016). These findings indicate that the 'dark' microglia could represent a subset of cells that become 
stressed under adaptive pressure, and as a result of their hyperactivity, leading to abnormal (or perhaps specialized) interactions with synapses. As previously discussed, synaptic loss is the best pathological correlate of cognitive decline across a myriad of neurological disorders associated with chronic psychosocial stress, including depression, schizophrenia, and neurodegenerative diseases (Duman and Aghajanian, 2012; Jurgens et al., 2016; McEwen et al., 2015; Spires-Jones and Hyman, 2014).

Microglial involvement with the gradual impairment of learning and memory during normal aging has been revealed by several studies. In particular, microglial priming by aging (Cornejo and von Bernhardi, 2016; Niraula et al., 2017), as characterized by a more proinflammatory basal state accompanied by increased expression of MHC class II and a decline in anti-inflammatory profile, is detrimental to neurogenesis, synaptic plasticity, as well as learning and memory (Ekdahl et al., 2003; McPherson et al., 2011; Vallieres et al., 2002). In addition, the aged brain, and especially the hippocampus, shows a decline in the levels of fractalkine (Bachstetter et al., 2011; Rogers et al., 2011) that may, in turn, affects healthy neuron-microglial crosstalk and hence contribute to the reduced synaptic plasticity and neurogenesis during normal aging (Sheridan and Murphy, 2013; Sierra et al., 2014).

\subsection{Microglial mediation of oxidative stress}

Apart from their mediation of inflammation and roles at synapses, microglia normally function as a redox-signaling center in the brain, which suggests additional mechanisms by which these cells could mediate neuronal damage upon psychosocial stress (and normal aging). Activation of two rate-limiting enzymes involved with the formation of L-kynurenine, tryptophan 2,3-dioxygenase (TDO) and IDO, was shown to increase kynurenine levels in the blood and cerebrospinal fluid (CSF) and to decrease serotonin production during depression in humans (Oxenkrug, 2010). Stress, corticosterone signaling and several inflammatory cytokines induced the IDO-kynurenine pathway in rodent and human microglial cells, resulting in the production of neurotoxic kynurenine metabolites, such as 3-hydroxy kynurenine and quinolinic acid, which in return could exacerbate inflammation, oxidative stress, as well as depressive behavior (reviewed in Capuron and Castanon, 2016; Dantzer et al., 2011). Likewise, administration of Lkynurenine to mice resulted in the emergence of a depression-like phenotype, while in LPS-treated mice, depression-like symptoms were attenuated by administration of either the IDO-antagonist 1-methyltryptophan (1 MT) or the microglial inhibitor minocycline (Dobos et al., 2012; Kiank et al., 2010; O'Connor et al., 2009).

Both rodent and human microglia express NADPH oxidase (NOX) 2 and NOX4 to regulate redox balance and stimulate oxidative stress upon neuronal injury or pathogen invasion, while NOX1 is exclusive to rodent microglia (Cheret et al., 2008; Cooney et al., 2013; Harrigan et al., 2008; Jadhav et al., 2014; Li et al., 2009; Mead et al., 2012). Neuronal injury increased microglial migration towards the damaged neurons (Heppner et al., 1998). As a neuroprotective mechanism, microglia generated inducible nitric oxide synthase (iNOS, NOS2) and release reactive oxygen species (ROS) and proteinases to clear neuronal debris (Banati et al., 1993). Each oxidase system works differently to regulate oxidative stress. NOX1 and NOX2 both contributed to LPS-induced microglial generation of intracellular superoxide $\left(\mathrm{O}_{2}{ }^{-}\right)$radical and $\mathrm{NO}$, while only NOX1 triggered microglial production of IL1 $\beta$ in vitro and in vivo in mice (Cheret et al., 2008). NOX1 deletion reduced microglial production of cytotoxic nitrite species and the loss of presynaptic proteins within the striatum of KO mice (Cheret et al., 2008). In addition, NOX2 was specifically upregulated in rodent microglia after traumatic brain injury and within the postmortem brain specimens of patients with CreutzfeldtJakob disease, a degenerative neurological disorder (Cooney et al., 2013; Sorce et al., 2014). Although NOX4 was not measured in rodent microglial cells after ischemic stroke (Kleinschnitz et al., 2010; Vallet et al., 2005), it was shown to be constitutively expressed in human microglia and to generate ROS as well as stimulate IL-6 expression ( Li et al.,
2009), suggesting that NOX subtypes contribute differently to the induction of oxidative stress in rodents $v s$ humans. For more information regarding the relationships between oxidative stress and inflammation in the context of mental disorders, our readers are referred to an excellent review on the topic (Miller and Raison, 2016).

\subsection{Aging effect on microglial oxidation (and inflammation) along with psychosocial stress}

Oxidative stress is measured in adults having experienced psychosocial stress or psychiatric diseases in childhood or at maturity. Adults with childhood abuse, war or divorce experience had shorter telomeres in blood cells due to glucocorticoid-associated oxidative stress damage (Ceccatelli et al., 2007; Schiavone et al., 2013; Tyrka et al., 2010), and increased susceptibility to ROS and brain cancers due to an elevation of ROS and NOS levels in the CNS (Bukhtoyarov and Samarin, 2009). Patients diagnosed with anxiety disorders, depression, schizophrenia or bipolar disorder also demonstrated decreased protein expressions of antioxidants $\mathrm{Cu}, \mathrm{Zn}$-superoxide dismutase (SOD1), glyoxalase (GLO)-1, glutathione reductase (GSR)-1 and glutathione, alongside increased immunoreactivities for the oxidative markers NOXs, DNA oxidation marker 8-hydroxy-2'-deoxyguanosine (8-oxo-dG), and exacerbated lipid peroxidation, within the cerebral cortex, hippocampus, and amygdala (Andreazza et al., 2008; Brown et al., 2014; Bruce-Keller et al., 2010; Gawryluk et al., 2011; Nunes et al., 2013; Sugama et al., 2016). Similar findings were also obtained in the blood and serum from patients having these psychiatric disorders (Altuntas et al., 2000; Bahceci et al., 2015; Kunz et al., 2008; Ranjekar et al., 2003; Zhang et al., 2006). On the other hand, reduced oxidative damage has been associated with resilience to psychosocial stress in humans (Aschbacher et al., 2013).

Similarly, in animals undergoing psychosocial stress, microglial phenotypic transformation was accompanied by exacerbated immunoreactivities against the antioxidants SOD1, GLO-1, and GSR-1, and the oxidative markers nitrotyrosine and NOXs, within the cerebral cortex, hippocampus, substantia nigra, and locus coeruleus (Gerecke et al., 2013; Nunes et al., 2013; Schiavone et al., 2009; Schiavone et al., 2012; Seo et al., 2012; Sugama et al., 2016; Vollert et al., 2011; Yoo et al., 2011). In addition, 7-weeks of social isolation was shown to exacerbate oxidative stress and NOX2 expression, increase the density of Iba $1^{+}$microglia, and the oxidative damage to neurons, in the nucleus accumbens and PFC, leading to enhance locomotor activity and impair memory (Schiavone et al., 2009). The relationship between microglial oxidative stress and inflammation has been characterized in vivo using ozone exposure, among several other paradigms, in rodents. For instance, ozone-depletion by the solvent 1-bromopropane and low-dose ozone exposure specifically increased oxidative stress and the density of Iba $1^{+}$microglia in the substantia nigra, leading to a progressive death of dopaminergic neurons, a hallmark feature of Parkinson's disease (PD) (Rivas-Arancibia et al., 2015). Upon ozone exposure, the neuroinflammation markers nuclear factor kappa B (NFKB) and cyclic oxygenase 2 (COX-2) were also upregulated alongside an overproduction of ROS (Rivas-Arancibia et al., 2015). Intracerebroventricular injection of thrombin, a coagulation factor acting as a potent microglial phenotypic modulator both in vitro and in vivo, similarly increased iNOS expression, $\mathrm{O}_{2}$-derived oxidant production, and protein oxidation, leading to neurotoxicity in the CA1 of rat hippocampus (Choi et al., 2005).

Several studies indicate that microglial impairment induced by psychosocial stress over the lifespan can accelerate brain aging and trigger the onset of neurodegenerative diseases that include AD and PD. Both psychological stress and aging potentiate oxidative stress (Miller and Sadeh, 2014; Rodrigues et al., 2014) and neuroinflammation (Cohen et al., 2012), which play together an important role in the pathogenesis of neurodegenerative diseases (Herrup, 2015; Kooi Ong et al., 2016; Ransohoff, 2016b). A mild restraint stressor that had little or no effects in young adult mice has been shown to disrupt spatial memory and 
elicit a significant neuroinflammatory response in aged mice, notably by triggering increased expressions of IL1 $\beta$ and MHC class II (Buchanan et al., 2008). Adult wild-type male mice undergoing repeated restraint stress ( 20 h per week for 6 weeks) further showed suppression of microglial neuroprotective functions, accompanied by a loss of dopaminergic neurons and the aggregation of $\alpha$-synuclein, another hallmark feature of PD, as a result of increased oxidative stress (Kooi Ong et al., 2016). Dopaminergic neurons constitute a population of cells highly vulnerable to the effects of oxidative stress, for various reasons that include not only their elevated mitochondrial bioenergetics and increased axonal arborization size (Pacelli et al., 2015), but also their dopaminergic content itself (Hastings et al., 1996; Kim et al., 2005; Offen et al., 1996).

Providing further support to this synergistic effect of inflammation and oxidative stress in the pathogenesis of neurodegenerative diseases, LPS injection in animals undergoing CUS also increased Iba ${ }^{+}$microglial density, pro-inflammatory markers expression and neuronal loss, and caused a prominent activation of stress-related signaling pathways in the rodent substantia nigra and hippocampus (de Pablos et al., 2014; Espinosa-Oliva et al., 2011). The mechanisms by which microglia trigger oxidative stress and inflammation-induced neuronal dysfunction and/or degeneration were recently summarized (Rojo et al., 2014; von Bernhardi et al., 2015). Upon aging and psychosocial stress, the reduced brain levels of anti-inflammatory and immunoregulatory factors shift the CNS microenvironment towards a pro-inflammatory state (Jurgens and Johnson, 2012). Microglial priming described in the aged brain (Kettenmann et al., 2011) additionally involves the recruitment of region-specific modifications at the transcriptional level (Grabert et al., 2016). In particular, several genes mediating immune cell phenotypic transformation and energy production were found to be upregulated during aging, with microglia from the cerebellum and hippocampus maintaining a more immune-alert state as compared to the microglia from striatum or cerebral cortex (Grabert et al., 2016).

\section{Proposed therapeutic targets to prevent stress- and aging-induced microglial impairment}

\subsection{Oxidative stress}

The above findings demonstrate the potential of using antioxidants as a therapeutic approach to suppress neuroinflammation and restore the redox balance of the brain in the context of aging and neurodegenerative diseases, which could also be utilized to prevent and/or treat neuropsychiatric disorders triggered by chronic psychosocial stress. Antioxidants were shown to inhibit inflammation, NO production, iNOS protein expression, intracellular ROS production/level and lipoxygenase activity induced by LPS in both BV2 and primary rodent microglial cells (Lee et al., 2016; Onasanwo et al., 2016; Salemme et al., 2016). In addition, antioxidants mediate their anti-inflammatory effects through upregulation of the anti-oxidative enzyme Heme oxygenase 1 (HO-1) and the oxidative enzyme inhibitor tissue metallopeptidase inhibitor 2 (TIMP2) via the Nrf2/ARE pathway in microglia (Lee et al., 2016; Onasanwo et al., 2016; Townsend and Johnson, 2016; Wang et al., 2016). A similar phenomenon was observed across in vivo studies. An antioxidant, biochanin A, suppressed the exaggerated Iba ${ }^{+}$microglial density, in addition to rescuing the neuronal loss and behavioral deficits in mouse models of AD and PD challenged with LPS (Justin Thenmozhi et al., 2016; Wang et al., 2015). Similarly, L-carnitine, a free radical scavenger, readily crossed the blood-brain barrier (Nalecz et al., 2004), reduced oxidative stress damage in brain tissues, and enhanced the functional outcomes in patients with mood disorders, neurometabolic disorders, or AD (Pettegrew et al., 2000; Ribas et al., 2014; Sitta et al., 2011). However, beneficial effects of antioxidants are still unclear and remain to be investigated in the context of psychosocial stress and neurodegenerative diseases (Ellwanger et al., 2016; Feng and Wang, 2012; Galasko et al., 2012; Mecocci and Polidori, 2012; Polidori and
Nelles, 2014). Encouragingly, inhibition of the oxidative stressneuroinflammatory cascade was shown to rescue the behavioral deficits associated with depression, bipolar disorder, and schizophrenia, in addition to restoring the redox balance in animal models (Deslauriers et al., 2014; Eren et al., 2007; Gawali et al., 2016; Jangra et al., 2016; Kulak et al., 2013; Masood et al., 2008; Ribeiro et al., 2013; Zugno et al., 2014) and humans (Berk et al., 2008; Galecki et al., 2009; Gautam et al., 2012; Khanzode et al., 2003; Lavoie et al., 2008; Magalhaes et al., 2016; Michalakeas et al., 2011; Ozcan et al., 2004; Reddy and Reddy, 2011).

\subsection{Neuron-microglia communication}

Unarguably, microglial pro-inflammatory properties should be restricted by using counter-regulatory mechanisms, while their homeostatic functions and synaptic interactions should be normalized to help cope with psychosocial stress. These functions are mainly regulated through bidirectional communication with neurons. Fractalkine signaling was found to exert anti-inflammatory properties almost two decades ago (Harrison et al., 1998). Intracerebroventricular injection of fractalkine-neutralizing antibodies greatly increased inflammatory response to TNF- $\alpha$ (Zujovic et al., 2001). Deficiency of $\mathrm{CX}_{3} \mathrm{CR} 1$ caused a massive phenotypic transformation of microglia upon repeated LPS injection (Cardona et al., 2006), increased IL-1 $\beta$ expression in the hippocampus, and worsened neurodegenerative progression in mouse models of AD (Bhaskar et al., 2010; Shaftel et al., 2007), although beneficial effects on neuronal survival were reported as well (Fuhrmann et al., 2010). Furthermore, profound impairment of hippocampaldependent learning and social interactions was detected in adult $\mathrm{CX}_{3} \mathrm{CR} 1-\mathrm{KO}$ animals, possibly due to altered synaptic pruning by microglia (Paolicelli et al., 2011; Zhan et al., 2014) or to an increased inflammation within the CNS (Rogers et al., 2011).

Studies in mice showed that deficiency in $\mathrm{CX}_{3} \mathrm{CR} 1$ or BDNF, as well as microglial depletion, similarly affected motor learning and fear conditioning (Parkhurst et al., 2013; Rogers et al., 2011). CX 3 CR1-deficiency also altered spatial learning and memory (Maggi et al., 2011; Rogers et al., 2011). Microglial depletion by using clodronate or oral administration of the inhibitor of colony-stimulating factor 1 receptor (CSF1R) transiently impaired spatial memory without disturbing social interactions when tested after 7 days of treatment (Torres et al., 2015). However, early microglial depletion by using clodronate liposomes (up to 70\% by postnatal day 6 ) had long-lasting consequences on social, moodrelated and locomotor behavior, when tested with the elevated plus maze and open field paradigms (Nelson and Lenz, 2017). Recently, a mouse model in which an activating enzyme that is essential for autophagy - Atg7 - was selectively defective in microglia, was similarly shown to display an increased density of dendritic spines and synaptic markers in the cerebral cortex, accompanied by impaired social and repetitive behaviors reminiscent of autism (Kim et al., 2016), suggesting that a defect of neuronal circuitry remodeling could be at cause.

Unexpectedly, $\mathrm{CX}_{3} \mathrm{CR} 1$-deficiency prevented the effects of CUS on microglial de-ramification and short- and long-term neuronal plasticity in the hippocampus CA1 region, as well as the emergence of depressivelike behavior (Milior et al., 2015). Furthermore, microglial phagocytosis of axon terminals and dendritic spines was basally elevated in the CA1, targeting synaptic elements in a non-specific manner, and was unmodulated by chronic stress (Milior et al., 2015). The $\mathrm{CX}_{3}$ CR1-deficient mice were also found to be resistant to stress-induced microglial hyperramification in the DG and to depression-like behavior and antidepressant treatment effect under the forced swim paradigm in another recent study (Hellwig et al., 2016). These findings indicate that microgliaregulated mechanisms could underlie the differential susceptibility to chronic stress and consequently the vulnerability to disorders, including major depression, triggered by stressful events.

Besides $\mathrm{CX}_{3} \mathrm{CL1}-\mathrm{CX}_{3} \mathrm{CR} 1$ signaling, several other molecular axes have been identified to mediate neuron-microglia interaction by targeting microglial receptors, including CD200R (Costello et al., 2011; 
Dentesano et al., 2014; Hoek et al., 2000; Meuth et al., 2008; Wright et al., 2000), sialic acid-binding immunoglobulin superfamily lectins (Siglecs) such as signal-regulatory-protein-alpha (SIRP $\alpha$ ) (Gitik et al., 2011; Linnartz and Neumann, 2013; Zhang et al., 2015), and TREM2 (Jiang et al., 2013; Takahashi et al., 2005; Walter, 2015). These receptors have been shown to prevent microglial transformation upon binding to their respective neuronal counterparts. Interaction between the neuronal ligand CD200 and microglial receptor CD200R was found to be essential for maintaining a healthy neuron-microglial crosstalk (Hoek et al., 2000; Neumann, 2001). Disruption of this interaction resulted in the emergence of microglial amoeboid morphologies associated with an increased expression of pro-inflammatory molecules in vitro (Zhang et al., 2011). Similarly, the constitutive relationships between neuronal CD47 and CD22 onto the microglial receptors SIRP- $\alpha$ and CD45 are considered to be important for controlling neuroinflammation (Gitik et al., 2011; Linnartz and Neumann, 2013; Zhang et al., 2015).

A number of secreted neuronal proteins were additionally shown to attenuate microglial phenotypic transformation, these include antiinflammatory cytokines such as TGF- $\beta$ (Boche et al., 2006; Brionne et al., 2003; Wahl et al., 2006) and IL-34 (Greter et al., 2012; Luo et al., 2013; Mizuno et al., 2011; Wang et al., 2012), neuropeptides (Delgado and Ganea, 2008; Reinke and Fabry, 2006; Waschek, 2013) and neurotrophins (Kerschensteiner et al., 2009; Neumann et al., 1998; Wei and Jonakait, 1999). Our own previous study also identified a neuronal adhesion molecule - ICAM-5 - that showed immune-dampening function too when cleaved from neurons by metalloproteinases (Tian et al., 2008). However, all of these studies were conducted under physiological or pathological conditions that are not strictly related to stress and anxiety.

\subsection{Neurotransmission and neuromodulation}

Several neurotransmitters, and especially monoamines, may represent potent target molecules to regulate microglial transformation and restore their physiological functions, as evidenced by drugs that modulate their synthesis, release, and recycling in both clinical studies and animal models of stress-related mental disorders, such as depression and anxiety. In patients suffering from acute depression, the elevated plasma levels of IL- 6 were reduced after treatments with fluoxetine, a selective serotonin reuptake inhibitor (SSRI) (Sluzewska et al., 1995). Recently, fluoxetine administered in an enriched environment was shown to increase the expression of pro-inflammatory markers in the hippocampus and in isolated microglia, while treatment in a stressful condition produced anti-inflammatory effects. In addition, these beneficial effects of fluoxetine in the stressful situation were accompanied by increased microglial cell body area, which is suggestive of an exacerbated transcriptional activity (perhaps anti-inflammatory), and by reduced process arborization area indicating that de-ramified morphologies are not exclusive to pro-inflammatory phenotypes (Alboni et al., 2016). In animal models, several anxiolytics and antidepressants have been reported to dampen neuroinflammation, as well as microglial morphological transformation in stressed animals. Fluoxetine was previously demonstrated to suppress the LPS-induced production of cytokines such as TNF- $\alpha$, IL-1 $\beta$, and IL- 6 by cultured microglial cells (Ha et al., 2006; Lim et al., 2009; Liu et al., 2011). Imipramine (a tricyclic antidepressant) inhibited IFN- $\gamma$-stimulated microglial production of IL-6 and NO (Hashioka et al., 2007) and TNF- $\alpha$ (Hwang et al., 2008) in vitro, and ameliorated the LPS-induced depression-like behaviors in rats in vivo (i.p., $10 \mathrm{mg} / \mathrm{kg}$ daily) (Yirmiya et al., 2001). Recently, Imipramine (i.p. or via drinking water, $20 \mathrm{mg} / \mathrm{kg}$ daily) was further shown to block microglial morphological transformation and pro-inflammatory gene expression in the brain under the CUS and repeated social defeat paradigms in mice (Kreisel et al., 2014; Ramirez et al., 2015).

It should be noted that the mechanisms underlying the immuneregulatory roles of these drugs can be multiple in the brain, e.g. despite that the drugs may have a direct influence on microglia, the possibility that their effects are achieved indirectly through modulation of neurons cannot be excluded. This is reinstated by a recent report showing that a $\gamma$-aminobutyric acid (GABA)-ergic anxiolytic and antidepressant clonazepam, a selective agonist of GABAA-R with high affinity for the central benzodiazepine binding site, which does not bind with high affinity to the TSPO expressed by microglia, blocked the accumulation of macrophages into the brain without affecting the production of proinflammatory cytokines in the spleen (Ramirez et al., 2016). GABA itself was shown to attenuate the pro-inflammatory response of microglial cultures (Kuhn et al., 2004). Additionally, receptors for acetylcholine and norepinephrine have been shown to be expressed by cultured microglial cells and therefore could regulate microglial functions as well (Blandino et al., 2009; Carnevale et al., 2007; McKim et al., 2015).

\section{Conclusion}

Heterogeneity in the individual response to psychosocial stress suggests that resilience is a complex neurobiological process that emerges

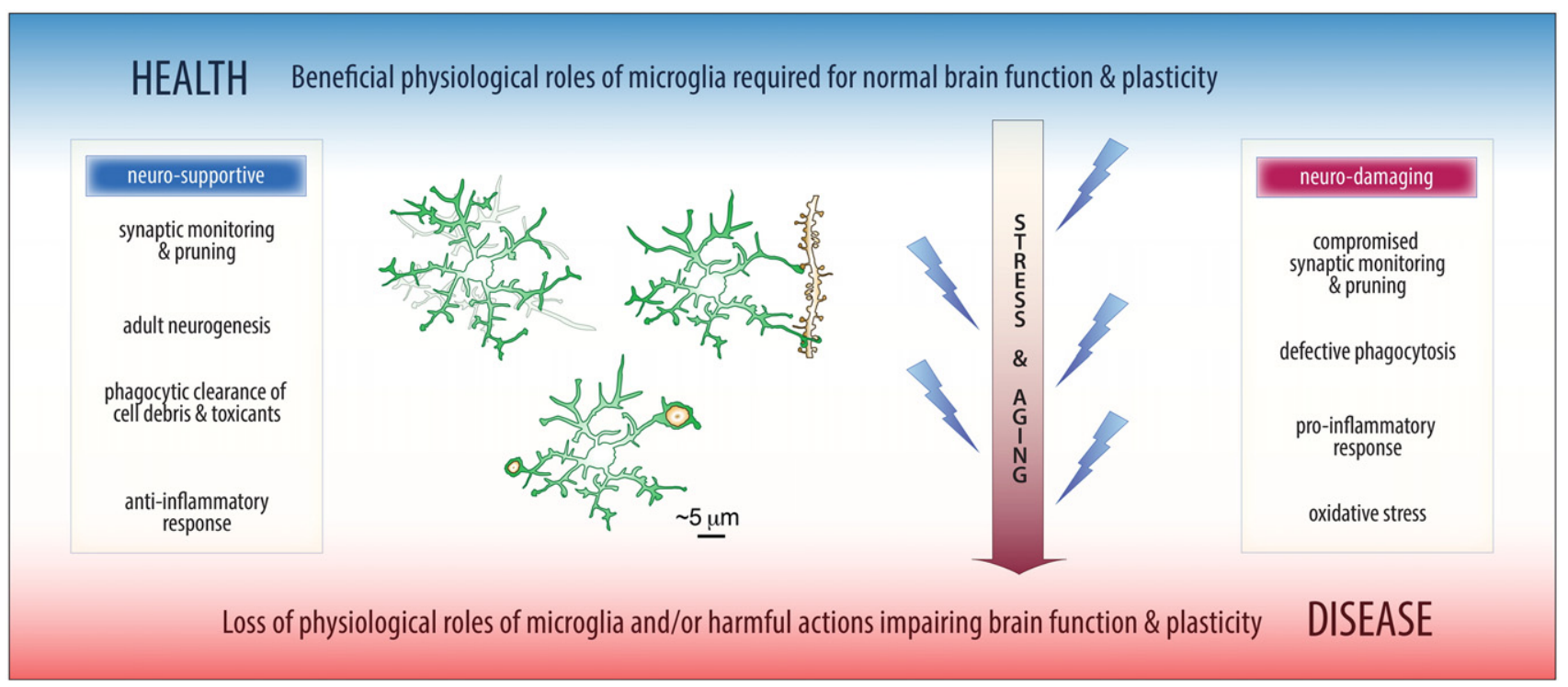

Fig. 1. Microglial dysfunction upon psychosocial stress and aging may have detrimental consequences on neuronal connectivity and behavior, thus contributing to brain diseases. 
from a multitude of gene-gene and gene-environment interactions. Several mechanisms were proposed to underlie the individual differences in stress resilience and vulnerability, involving the interactions of immune cells and their inflammatory mediators with various neurotransmitter and other neuromodulatory systems (Pfau and Russo, 2015).

The integrative network formed by neurons and (micro)glia plays a major role in mediating the normal adult brain functions. Here, we have recounted current advances in this research field and discussed mechanisms underlying microglial regulation of neuronal synapses and conversely, the neuronal regulation of microglial phenotypic transformation at morphological and molecular levels. From the above discussions, one can see that microglia (as well as other immune and glial cells that are not covered by us here) are pivotal for actively shaping neuronal structural and functional plasticity. The responsible mechanisms include the clearance of cellular debris and toxicants, neurogenesis, antiinflammatory response, as well as synaptic monitoring and pruning (see Fig. 1 for a schematic representation). Reciprocally, neurons play important roles in nurturing microglia and maintaining their inflammatory gene production, oxidative stress response, and phagocytosis at an appropriate level, in order to keep their own homeostasis under normal physiological conditions. However, both neurons and microglia are sensitive to psychosocial stress and persistent stress may compromise the dynamic balance of neuron-microglia interaction, disturb synaptic neurotransmission and hence increase the susceptibility to mental disorders.

Similar to chronic psychosocial stress, the aging process also potentiates oxidative stress and neuroinflammation, leading to compromised synaptic monitoring and pruning functions, notably through a defective phagocytosis (see Fig. 1). Chronic psychological stress is an environmental risk factor well known for accelerating aging, predisposing to neuropsychiatric and neurodegenerative diseases, as well as accelerating their progression, and exacerbating their symptoms. If depression is the devastating outcome of psychosocial stress, a considered risk factor and a comorbidity in neurodegenerative diseases, stress resilience, on the other hand, is the positive outcome of stress adaptation associated with healthy aging (Charney, 2004; Pfau and Russo, 2015). Since microglia act as a critical cellular component in the stress response, therapeutic interventions promoting resilience of these immune cells to aging and psychosocial stress could allow delaying the onset and/or progression of mental disorders.

$\begin{array}{ll}\text { Abbreviations } \\ \text { ACC } & \text { anterior cingulate cortex } \\ \text { BDNF } & \text { brain-derived neurotrophic factor } \\ \text { CNS } & \text { central nervous system } \\ \text { CSF } & \text { cerebrospinal fluid } \\ \text { CUS } & \text { chronic unpredictable stress } \\ \text { DG } & \text { dentate gyrus } \\ \text { GABA } & \gamma \text {-aminobutyric acid } \\ \text { IDO } & \text { indoleamine-2,3-dioxygenase } \\ \text { IFN } & \text { interferon } \\ \text { IL } & \text { interleukin } \\ \text { KO } & \text { knock-out } \\ \text { LPS } & \text { lipopolysaccharide } \\ \text { LBP } & \text { lipopolysaccharide-binding protein } \\ \text { MHC } & \text { major histocompatibility complex } \\ \text { NOS } & \text { nitric oxide synthase } \\ \text { PFC } & \text { prefrontal cortex } \\ \text { ROS } & \text { reducing oxidative species } \\ \text { Siglec } & \text { sialic acid-binding immunoglobulin superfamily lectin } \\ \text { SIRP } \alpha & \text { signal-regulatory-protein-alpha } \\ \text { TGF- } \beta & \text { transforming growth factor-beta } \\ \text { TNF } & \text { tumor necrosis factor } \\ \text { TREM } & \text { triggering receptor expressed on myeloid cells } \\ \text { TSPO } & \text { translocator protein }\end{array}$

\section{Acknowledgements}

LT is funded by the Academy of Finland projects No. 1273108 and 1283085, and European Commission FP7/Cooperation subprogramme/HEALTH-2013-Innovation Grant No. 602919. YLT is funded by the International (Regional) Cooperation Project of the National Natural Science Foundation of China (81461130016), the High-level health-technology personnel in Beijing healthcare system (2014-3097), the National Natural Science Foundation of China (81371477 \& 81000509), and the Beijing Municipal Natural Science Foundation (7132063 and 7072035). XYZ is funded by the NARSAD Independent Investigator Grant (20314). MET is funded by the Natural Sciences and Engineering Research Council of Canada (NSERC) RGPIN-2014-05308. $\mathrm{KB}$ is recipient of an excellence scholarship from the CHU de Québec Foundation.

\section{References}

Adelson, J.D., et al., 2016. Developmental sculpting of intracortical circuits by MHC class I $\mathrm{H} 2-\mathrm{Db}$ and H2-Kb. Cereb. Cortex 26, 1453-1463.

Alboni, S., et al., 2016. Fluoxetine treatment affects the inflammatory response and microglial function according to the quality of the living environment. Brain Behav. Immun. 58, 261-271

Alkadhi, K.A., 2012. Chronic psychosocial stress exposes Alzheimer's disease phenotype in a novel at-risk model. Front. Biosci. (Elite Ed.) 4, 214-229.

Alkadhi, K.A., Tran, T.T., 2015. Chronic stress decreases basal levels of memory-related signaling molecules in area CA1 of at-risk (subclinical) model of Alzheimer's disease. Mol. Neurobiol. 52, 93-100.

Altuntas, I., et al., 2000. Erythrocyte superoxide dismutase and glutathione peroxidase activities, and malondialdehyde and reduced glutathione levels in schizophrenic patients. Clin. Chem. Lab. Med. 38, 1277-1281.

Andreazza, A.C., et al., 2008. Oxidative stress markers in bipolar disorder: a meta-analysis. J. Affect. Disord. 111, 135-144.

Arnoux, I., Audinat, E., 2015. Fractalkine signaling and microglia functions in the developing brain. Neural Plast. 2015, 689404

Aschbacher, K., et al., 2013. Good stress, bad stress and oxidative stress: insights from anticipatory cortisol reactivity. Psychoneuroendocrinology 38, 1698-1708.

Bachstetter, A.D., et al., 2011. Fractalkine and CX 3 CR1 regulate hippocampal neurogenesis in adult and aged rats. Neurobiol. Aging 32, 2030-2044.

Bahceci, B., et al., 2015. Prolidase activity and oxidative stress in patients with schizophrenia: a preliminary study. J. Pak. Med. Assoc. 65, 131-135.

Banati, R.B., et al., 1993. Cytotoxicity of microglia. Glia 7, 111-118.

Bellinger, D.L., Lorton, D., 2014. Autonomic regulation of cellular immune function. Auton. Neurosci. 182, 15-41.

Berk, M., et al., 2008. N-acetyl cysteine as a glutathione precursor for schizophrenia-a double-blind, randomized, placebo-controlled trial. Biol. Psychiatry 64, 361-368.

Bhaskar, K., et al., 2010. Regulation of tau pathology by the microglial fractalkine receptor. Neuron 68, 19-31.

Biber, K., et al., 2016. Central nervous system myeloid cells as drug targets: current status and translational challenges. Nat. Rev. Drug Discov. 15, 110-124.

Bilbo, S.D., Schwarz, J.M., 2012. The immune system and developmental programming of brain and behavior. Front. Neuroendocrinol. 33, 267-286.

Bisht, K., et al., 2016. Dark microglia: A new phenotype predominantly associated with pathological states. Glia.

Blandino Jr., P., et al., 2009. Gene expression changes in the hypothalamus provide evidence for regionally-selective changes in IL- 1 and microglial markers after acute stress. Brain Behav. Immun. 23, 958-968.

Bloomfield, P.S., et al., 2016. Microglial activity in people at ultra high risk of psychosis and in schizophrenia: an [(11)C]PBR28 PET brain imaging study. Am. J. Psychiatry 173, 44-52.

Boche, D., et al., 2006. TGFbeta1 regulates the inflammatory response during chronic neurodegeneration. Neurobiol. Dis. 22, 638-650.

Brionne, T.C., et al., 2003. Loss of TGF-beta 1 leads to increased neuronal cell death and microgliosis in mouse brain. Neuron 40,1133-1145.

Brown, N.C., Andreazza, A.C., Young, L.T., 2014. An updated meta-analysis of oxidative stress markers in bipolar disorder. Psychiatry Res. 218, 61-68.

Bruce-Keller, A.J., et al., 2010. NOX activity is increased in mild cognitive impairment. Antioxid. Redox Signal. 12, 1371-1382.

Buchanan, J.B., et al., 2008. Cognitive and neuroinflammatory consequences of mild repeated stress are exacerbated in aged mice. Psychoneuroendocrinology 33, 755-765.

Bukhtoyarov, O.V., Samarin, D.M., 2009. Psychogenic carcinogenesis: carcinogenesis is without exogenic carcinogens. Med. Hypotheses 73, 531-536.

Burke, N.N., et al., 2014. Minocycline modulates neuropathic pain behaviour and cortical M1-M2 microglial gene expression in a rat model of depression. Brain Behav. Immun. 42, $147-156$

Calcia, M.A., et al., 2016. Stress and neuroinflammation: a systematic review of the effects of stress on microglia and the implications for mental illness. Psychopharmacology (Berl)

Capuron, L., Castanon, N., 2016. Role of inflammation in the development of neuropsychiatric symptom domains: evidence and mechanisms. Curr. Top. Behav. Neurosci.

Cardona, A.E., et al., 2006. Control of microglial neurotoxicity by the fractalkine receptor. Nat. Neurosci. 9, 917-924.

Carnevale, D., De Simone, R., Minghetti, L., 2007. Microglia-neuron interaction in inflammatory and degenerative diseases: role of cholinergic and noradrenergic systems. CNS Neurol. Disord. Drug Targets 6, 388-397. 
Castanon, N., Luheshi, G., Laye, S., 2015. Role of neuroinflammation in the emotional and cognitive alterations displayed by animal models of obesity. Front. Neurosci. 9, 229.

Ceccatelli, S., et al., 2007. Mechanisms and modulation of neural cell damage induced by oxidative stress. Physiol. Behav. 92, 87-92.

Chabry, J., et al., 2015. Enriched environment decreases microglia and brain macrophages inflammatory phenotypes through adiponectin-dependent mechanisms: Relevance to depressive-like behavior. Brain Behav. Immun. 50, 275-287.

Charney, D.S., 2004. Psychobiological mechanisms of resilience and vulnerability: implications for successful adaptation to extreme stress. Am. J. Psychiatry 161, 195-216.

Cheret, C., et al., 2008. Neurotoxic activation of microglia is promoted by a nox1dependent NADPH oxidase. J. Neurosci. 28, 12039-12051.

Choi, S.H., et al., 2005. Thrombin-induced oxidative stress contributes to the death of hippocampal neurons in vivo: role of microglial NADPH oxidase. J. Neurosci. 25, 4082-4090.

Cohen, S., et al., 2012. Chronic stress, glucocorticoid receptor resistance, inflammation, and disease risk. Proc. Natl. Acad. Sci. U. S. A. 109, 5995-5999.

Cooney, S.J., Bermudez-Sabogal, S.L., Byrnes, K.R., 2013. Cellular and temporal expression of NADPH oxidase (NOX) isotypes after brain injury. J. Neuroinflammation 10, 155.

Cornejo, F., von Bernhardi, R., 2016. Age-dependent changes in the activation and regulation of microglia. Adv. Exp. Med. Biol. 949, 205-226.

Costello, D.A., et al., 2011. Long term potentiation is impaired in membrane glycoprotein CD200-deficient mice: a role for Toll-like receptor activation. J. Biol. Chem. 286 34722-34732.

Couch, Y., et al., 2013. Microglial activation, increased TNF and SERT expression in the prefrontal cortex define stress-altered behaviour in mice susceptible to anhedonia. Brain Behav. Immun. 29, 136-146.

Cunningham, C.L., Martinez-Cerdeno, V., Noctor, S.C., 2013. Microglia regulate the number of neural precursor cells in the developing cerebral cortex. J. Neurosci. 33, 4216-4233.

D'Agostino, P.M., et al., 2012. Brain dendritic cells: biology and pathology. Acta Neuropathol. 124, 599-614.

Dantzer, R., et al., 2011. Inflammation-associated depression: from serotonin to kynurenine. Psychoneuroendocrinology 36, 426-436.

Davalos, D., et al., 2005. ATP mediates rapid microglial response to local brain injury in vivo. Nat. Neurosci. 8, 752-758.

de Pablos, R.M., et al., 2014. Chronic stress enhances microglia activation and exacerbates death of nigral dopaminergic neurons under conditions of inflammation. J. Neuroinflammation 11, 34

Delgado, M., Ganea, D., 2008. Anti-inflammatory neuropeptides: a new class of endogenous immunoregulatory agents. Brain Behav. Immun. 22, 1146-1151.

Delpech, J.C., et al., 2015a. Microglia in neuronal plasticity: Influence of stress. Neuropharmacology 96, 19-28.

Delpech, J.C., et al., 2015b. Microglial activation enhances associative taste memory through purinergic modulation of glutamatergic neurotransmission. J. Neurosci. 35 , 3022-3033.

Dentesano, G., et al., 2014. CD200R1 and CD200 expression are regulated by PPARgamma in activated glial cells. Glia 62, 982-998.

Deslauriers, J., et al., 2014. Preventive effect of alpha-lipoic acid on prepulse inhibition deficits in a juvenile two-hit model of schizophrenia. Neuroscience 272, 261-270.

Diz-Chaves, Y., et al., 2012. Prenatal stress causes alterations in the morphology of microglia and the inflammatory response of the hippocampus of adult female mice. J. Neuroinflammation 9, 71

Duman, R.S., Aghajanian, G.K., 2012. Synaptic dysfunction in depression: potential therapeutic targets. Science $338,68-72$

Dungrawala, H., Manukyan, A., Schneider, B.L., 2010. Gene regulation: global transcription rates scale with size. Curr. Biol. 20, R979-R981.

Ekdahl, C.T., et al., 2003. Inflammation is detrimental for neurogenesis in adult brain. Proc. Natl. Acad Sci. U. S. A. 100, 13632-13637.

Ellwanger, J.H., et al., 2016. Biological functions of selenium and its potential influence on Parkinson's disease. An. Acad. Bras. Cienc

Eren, I., Naziroglu, M., Demirdas, A., 2007. Protective effects of lamotrigine, aripiprazole and escitalopram on depression-induced oxidative stress in rat brain. Neurochem. Res. 32, 1188-1195.

Espinosa-Oliva, A.M., et al., 2011. Stress is critical for LPS-induced activation of microglia and damage in the rat hippocampus. Neurobiol. Aging 32, 85-102.

Feng, Y., Wang, X., 2012. Antioxidant therapies for Alzheimer's disease. Oxid. Med. Cell. Longev. 2012, 472932.

Fidler, J.A., et al., 2011. Disease progression in a mouse model of amyotrophic lateral sclerosis: the influence of chronic stress and corticosterone. FASEB J. 25, 4369-4377.

Fourgeaud, L., et al., 2016. TAM receptors regulate multiple features of microglial physiology. Nature 532, 240-244.

Franco, R., Fernandez-Suarez, D., 2015. Alternatively activated microglia and macrophages in the central nervous system. Prog. Neurobiol. 131, 65-86.

Frank, M.G., et al., 2007. Microglia serve as a neuroimmune substrate for stress-induced potentiation of CNS pro-inflammatory cytokine responses. Brain Behav. Immun. 21, 47-59.

Fuhrmann, M., et al., 2010. Microglial Cx3cr1 knockout prevents neuron loss in a mouse model of Alzheimer's disease. Nat. Neurosci. 13, 411-413.

Galasko, D.R., et al., 2012. Antioxidants for Alzheimer disease: a randomized clinical trial with cerebrospinal fluid biomarker measures. Arch. Neurol. 69, 836-841.

Galecki, P., et al., 2009. Relation between functional polymorphism of catalase gene $(-262 \mathrm{C}>\mathrm{T})$ and recurrent depressive disorder. Neuro Endocrinol. Lett. 30, 357-362.

Gautam, M., et al , 2012. Role of antioxidants in generalised anxiety disorder and depression. Indian J. Psychiatry 54, 244-247.

Gawali, N.B., et al., 2016. Agmatine ameliorates lipopolysaccharide induced depressivelike behaviour in mice by targeting the underlying inflammatory and oxidonitrosative mediators. Pharmacol. Biochem. Behav. 149, 1-8.

Gawryluk, J.W., et al., 2011. Decreased levels of glutathione, the major brain antioxidant, in post-mortem prefrontal cortex from patients with psychiatric disorders. Int. J. Neuropsychopharmacol. 14, 123-130.

Gerecke, K.M., et al., 2013. Exercise protects against chronic restraint stress-induced oxidative stress in the cortex and hippocampus. Brain Res. 1509, 66-78.
Ginhoux, F., et al., 2010. Fate mapping analysis reveals that adult microglia derive from primitive macrophages. Science 330, 841-845.

Giovanoli, S., et al., 2013. Stress in puberty unmasks latent neuropathological consequences of prenatal immune activation in mice. Science 339, 1095-1099.

Gitik, M., et al., 2011. Myelin down-regulates myelin phagocytosis by microglia and macrophages through interactions between CD47 on myelin and SIRPalpha (signal regulatory protein-alpha) on phagocytes. J. Neuroinflammation 8, 24.

Gomez-Gonzalez, B., Escobar, A., 2010. Prenatal stress alters microglial development and distribution in postnatal rat brain. Acta Neuropathol. 119, 303-315.

Grabert, K., et al., 2016. Microglial brain region-dependent diversity and selective regional sensitivities to aging. Nat. Neurosci. 19, 504-516.

Greter, M., et al., 2012. Stroma-derived interleukin-34 controls the development and maintenance of Langerhans cells and the maintenance of microglia. Immunity 37 $1050-1060$.

Ha, E., et al., 2006. Fluoxetine increases the nitric oxide production via nuclear facto kappa B-mediated pathway in BV2 murine microglial cells. Neurosci. Lett. 397, 185-189.

Haarman, B.C., et al., 2014. Neuroinflammation in bipolar disorder - a [(11)C]-(R)PK11195 positron emission tomography study. Brain Behav. Immun. 40, 219-225.

Hanamsagar, R., Bilbo, S.D., 2016. Sex differences in neurodevelopmental and neurodegenerative disorders: Focus on microglial function and neuroinflammation during development. J. Steroid Biochem. Mol. Biol. 160, 127-133.

Hanisch, U.K., Kettenmann, H., 2007. Microglia: active sensor and versatile effector cells in the normal and pathologic brain. Nat. Neurosci. 10, 1387-1394.

Harrigan, T.J., et al., 2008. Activation of microglia with zymosan promotes excitatory amino acid release via volume-regulated anion channels: the role of NADPH oxidases. J. Neurochem. 106, 2449-2462.

Harrison, J.K., et al., 1998. Role for neuronally derived fractalkine in mediating interaction between neurons and CX3CR1-expressing microglia. Proc. Natl. Acad. Sci. U. S. A. 95 10896-10901.

Hashioka, S., et al., 2007. Antidepressants inhibit interferon-gamma-induced microglial production of IL-6 and nitric oxide. Exp. Neurol. 206, 33-42.

Hastings, T.G., Lewis, D.A., Zigmond, M.J., 1996. Role of oxidation in the neurotoxic effects of intrastriatal dopamine injections. Proc. Natl. Acad. Sci. U. S. A. 93 1956-1961.

Haynes, S.E., et al., 2006. The P2Y(12) receptor regulates microglial activation by extracelular nucleotides. Nat. Neurosci. 9, 1512-1519.

Hellwig, S., et al., 2016. Altered microglia morphology and higher resilience to stressinduced depression-like behavior in CX3CR1-deficient mice. Brain Behav. Immun. $55,126-137$

Heppner, F.L., et al., 1998. Activated microglial cells migrate towards sites of excitotoxic neuronal injury inside organotypic hippocampal slice cultures. Eur. J. Neurosci. 10, $3284-3290$

Herrup, K., 2015. The case for rejecting the amyloid cascade hypothesis. Nat. Neurosci. 18 , 794-799.

Hinwood, M., et al., 2012. Evidence that microglia mediate the neurobiological effects of chronic psychological stress on the medial prefrontal cortex. Cereb. Cortex 22, $1442-1454$.

Hinwood, M., et al., 2013. Chronic stress induced remodeling of the prefrontal cortex: tructural re-organization of microglia and the inhibitory effect of minocycline. Cereb. Cortex 23, 1784-1797.

Hoek, R.M., et al., 2000. Down-regulation of the macrophage lineage through interaction with OX2 (CD200). Science 290, 1768-1771.

Hong, S., Stevens, B., 2016. Microglia: phagocytosing to clear, sculpt, and eliminate. Dev. Cell 38, 126-128.

Hong, S., Dissing-Olesen, L., Stevens, B., 2016. New insights on the role of microglia in synaptic pruning in health and disease. Curr. Opin. Neurobiol. 36, 128-134.

Hoshiko, M., et al., 2012. Deficiency of the microglial receptor CX3CR1 impairs postnata functional development of thalamocortical synapses in the barrel cortex J. Neurosci. 32, 15106-15111.

Hou, G., et al., 2014. Chronic stress and Parkinson's disease. CNS Neurosci. Ther. 20, 1-2

Hovatta, I., Barlow, C., 2008. Molecular genetics of anxiety in mice and men. Ann. Med. 40 92-109.

Hovatta, I., et al., 2005. Glyoxalase 1 and glutathione reductase 1 regulate anxiety in mice. Nature 438, 662-666.

Hwang, J., et al., 2008. Inhibition of glial inflammatory activation and neurotoxicity by tricyclic antidepressants. Neuropharmacology 55, 826-834.

Iwata, M., et al., 2016. Psychological stress activates the inflammasome via release of adenosine triphosphate and stimulation of the purinergic type 2 X7 receptor. Biol. Psychiatry $80,12-22$

Jadhav, V.S., Krause, K.H., Singh, S.K., 2014. HIV-1 Tat C modulates NOX2 and NOX4 expressions through miR-17 in a human microglial cell line. J. Neurochem. 131, 803-815.

Jangra, A., Sriram, C.S., Lahkar, M., 2016. Lipopolysaccharide-induced behavioral alterations are alleviated by sodium phenylbutyrate via attenuation of oxidative stress and neuroinflammatory cascade. Inflammation 39, 1441-1452.

Jenmalm, M.C., et al., 2006. Regulation of myeloid cell function through the CD200 receptor. J. Immunol. 176, 191-199.

Jiang, T., et al., 2013. TREM2 in Alzheimer's disease. Mol. Neurobiol. 48, 180-185.

Johnson, J.D., Zimomra, Z.R., Stewart, L.T., 2013. Beta-adrenergic receptor activation primes microglia cytokine production. J. Neuroimmunol. 254, 161-164.

Jurgens, H.A., Johnson, R.W., 2012. Dysregulated neuronal-microglial cross-talk during aging. Stress Inflamm. 233, 40-48.

Jurgens, T., et al., 2016. Reconstruction of single cortical projection neurons reveals primary spine loss in multiple sclerosis. Brain 139, 39-46.

Justin Thenmozhi, A., et al., 2016. Hesperidin ameliorates cognitive dysfunction, oxidative stress and apoptosis against aluminium chloride induced rat model of Alzheimer's disease. Nutr. Neurosci.

Kerschensteiner, M., Meinl, E., Hohlfeld, R., 2009. Neuro-immune crosstalk in CNS diseases. Neuroscience 158, 1122-1132.

Kettenmann, H., et al., 2011. Physiology of microglia. Physiol. Rev. 91, 461-553. 
Kettenmann, H., Kirchhoff, F., Verkhratsky, A., 2013. Microglia: new roles for the synaptic stripper. Neuron $77,10-18$

Khanzode, S.D., et al., 2003. Oxidative damage and major depression: the potential antioxidant action of selective serotonin re-uptake inhibitors. Redox Rep. 8, 365-370.

Kierdorf, K., et al., 2013. Microglia emerge from erythromyeloid precursors via Pu.1- and Irf8-dependent pathways. Nat. Neurosci. 16, 273-280.

Kim, S.T., et al., 2005. Immobilization stress causes increases in tetrahydrobiopterin, dopamine, and neuromelanin and oxidative damage in the nigrostriatal system. J. Neurochem. 95, 89-98.

Kim, H.J., et al., 2016. Deficient autophagy in microglia impairs synaptic pruning and causes social behavioral defects. Mol. Psychiatry.

Kleinschnitz, C., et al., 2010. Post-stroke inhibition of induced NADPH oxidase type 4 prevents oxidative stress and neurodegeneration. PLoS Biol. 8.

Knuesel, I., et al., 2014. Maternal immune activation and abnormal brain development across CNS disorders. Nat. Rev. Neurol. 10, 643-660.

Kooi Ong, L., et al., 2016. Reconsidering the role of glial cells in chronic stress-induced dopaminergic neurons loss within the substantia nigra? Friend or foe? Brain Behav. Immun.

Kopp, B.L., Wick, D., Herman, J.P., 2013. Differential effects of homotypic vs. heterotypic chronic stress regimens on microglial activation in the prefrontal cortex. Physiol. Behav. 122, 246-252.

Kreisel, T., et al., 2014. Dynamic microglial alterations underlie stress-induced depressivelike behavior and suppressed neurogenesis. Mol. Psychiatry 19, 699-709.

Kuhn, S.A., et al., 2004. Microglia express GABA(B) receptors to modulate interleukin release. Mol. Cell. Neurosci. 25, 312-322.

Kulak, A., et al., 2013. Redox dysregulation in the pathophysiology of schizophrenia and bipolar disorder: insights from animal models. Antioxid. Redox Signal. 18, $1428-1443$.

Kulesskaya, N., et al., 2014. Mixed housing with DBA/2 mice induces stress in C57BL/6 mice: implications for interventions based on social enrichment. Front. Behav Neurosci. 8, 257

Kunz, M., et al., 2008. Elevated serum superoxide dismutase and thiobarbituric acid reactive substances in different phases of bipolar disorder and in schizophrenia. Prog. Neuropsychopharmacol. Biol. Psychiatry 32, 1677-1681.

Laskaris, L.E., et al., 2016. Microglial activation and progressive brain changes in schizophrenia. Br. J. Pharmacol. 173, 666-680.

Lavoie, S., et al., 2008. Glutathione precursor, N-acetyl-cysteine, improves mismatch negativity in schizophrenia patients. Neuropsychopharmacology 33, 2187-2199.

Lee, H., et al., 2014. Synapse elimination and learning rules co-regulated by MHC class 1 H2-Db. Nature 509, 195-200.

Lee, Y.Y., et al., 2016. Anti-inflammatory and antioxidant mechanism of tangeretin in activated microglia. J. Neuroimmune Pharmacol. 11, 294-305.

Lehmann, M.L., et al., 2016. Social defeat induces depressive-like states and microglial activation without involvement of peripheral macrophages. J. Neuroinflammation 13 , 224.

Lemaire, V., et al., 2000. Prenatal stress produces learning deficits associated with an inhibition of neurogenesis in the hippocampus. Proc. Natl. Acad. Sci. U. S. A. 97 $11032-11037$

Lemke, G., 2013. Biology of the TAM receptors. Cold Spring Harb. Perspect. Biol. 5 , a009076.

Li, B., et al., 2009. NOX4 expression in human microglia leads to constitutive generation of reactive oxygen species and to constitutive IL-6 expression. J. Innate Immun. 1 , 570-581.

Li, Z., et al., 2014. Microglia are polarized to M1 type in high-anxiety inbred mice in response to lipopolysaccharide challenge. Brain Behav. Immun. 38, 237-248.

Lim, C.M., et al., 2009. Fluoxetine affords robust neuroprotection in the postischemic brain via its anti-inflammatory effect. J. Neurosci. Res. 87, 1037-1045.

Linnartz, B., Neumann, H., 2013. Microglial activatory (immunoreceptor tyrosinebased activation motif)- and inhibitory (immunoreceptor tyrosine-based inhibition motif)-signaling receptors for recognition of the neuronal glycocalyx. Glia $61,37-46$.

Liston, C., Gan, W.B., 2011. Glucocorticoids are critical regulators of dendritic spine development and plasticity in vivo. Proc. Natl. Acad. Sci. U. S. A. 108, 16074-16079.

Liu, D., et al., 2011. Anti-inflammatory effects of fluoxetine in lipopolysaccharide(LPS)stimulated microglial cells. Neuropharmacology 61, 592-599.

Luo, J., et al., 2013. Colony-stimulating factor 1 receptor (CSF1R) signaling in injured neurons facilitates protection and survival. J. Exp. Med. 210, 157-172.

Ma, L., et al., 2015a. Differential expression of brain immune genes and schizophreniarelated behavior in C57BL/6N and DBA/2J female mice. Psychiatry Res. 226, 211-216.

Ma, L., et al., 2015b. Association of brain immune genes with social behavior of inbred mouse strains. J. Neuroinflammation 12, 75 .

Machado, A., et al., 2014. Chronic stress as a risk factor for Alzheimer's disease. Rev. Neurosci. 25, 785-804

Magalhaes, P.V., et al., 2016. Antioxidant treatments for schizophrenia. Cochrane Database Syst. Rev. 2, Cd008919.

Maggi, L., et al., 2009. LTP impairment by fractalkine/CX3CL1 in mouse hippocampus is mediated through the activity of adenosine receptor type 3 (A3R). J. Neuroimmunol. 215, 36-42.

Maggi, L., et al., 2011. CX(3)CR1 deficiency alters hippocampal-dependent plasticity phenomena blunting the effects of enriched environment. Front. Cell. Neurosci. 5, 22.

Martelli, D., et al., 2014. Reflex control of inflammation by sympathetic nerves, not the vagus. J. Physiol. 592, 1677-1686.

Masood, A., et al., 2008. Reversal of oxidative stress-induced anxiety by inhibition of phosphodiesterase-2 in mice. J. Pharmacol. Exp. Ther. 326, 369-379.

McEwen, B.S., Gould, E.A., Sakai, R.R., 1992. The vulnerability of the hippocampus to protective and destructive effects of glucocorticoids in relation to stress. Br. J. Psychiatry Suppl. 18-23.

McEwen, B.S., et al., 2015. Mechanisms of stress in the brain. Nat. Neurosci. 18 1353-1363.

McKim, D.B., et al., 2015. Sympathetic release of splenic monocytes promotes recurring anxiety following repeated social defeat. Biol. Psychiatry.
McKim, D.B., et al., 2016. Neuroinflammatory dynamics underlie memory impairments after repeated social defeat. 36, 2590-2604.

McPherson, C.A., Aoyama, M., Harry, G.J., 2011. Interleukin (IL)-1 and IL-6 regulation of neural progenitor cell proliferation with hippocampal injury: differential regulatory pathways in the subgranular zone (SGZ) of the adolescent and mature mouse brain. Brain Behav. Immun. 25, 850-862.

Mead, E.L., et al., 2012. Microglial neurotransmitter receptors trigger superoxide production in microglia; consequences for microglial-neuronal interactions. J. Neurochem. 121, 287-301.

Mecocci, P., Polidori, M.C., 2012. Antioxidant clinical trials in mild cognitive impairment and Alzheimer's disease. Biochim. Biophys. Acta 1822, 631-638.

Meuth, S.G., et al., 2008. CNS inflammation and neuronal degeneration is aggravated by impaired CD200-CD200R-mediated macrophage silencing. J. Neuroimmunol. 194, 62-69.

Michalakeas, C.A., et al., 2011. Effects of sertraline on circulating markers of oxidative stress in depressed patients with chronic heart failure: a pilot study. J. Card. Fail. $17,748-754$

Milior, G., et al., 2015. Fractalkine receptor deficiency impairs microglial and neuronal responsiveness to chronic stress. Brain Behav. Immun.

Miller, A.H., Raison, C.L., 2016. The role of inflammation in depression: from evolutionary imperative to modern treatment target. Nat. Rev. Immunol. 16, 22-34.

Miller, M.W., Sadeh, N., 2014. Traumatic stress, oxidative stress and post-traumatic stress disorder: neurodegeneration and the accelerated-aging hypothesis. Mol. Psychiatry $19,1156-1162$.

Mizuno, T., et al., 2011. Interleukin-34 selectively enhances the neuroprotective effects of microglia to attenuate oligomeric amyloid-beta neurotoxicity. Am. J. Pathol. 179, 2016-2027.

Mo, C., Renoir, T., Hannan, A.J., 2014. Effects of chronic stress on the onset and progression of Huntington's disease in transgenic mice. Neurobiol. Dis. 71, 81-94.

Monif, M., et al., 2009. The P2X7 receptor drives microglial activation and proliferation: a trophic role for P2X7R pore. J. Neurosci. 29, 3781-3791.

Morgan, J.T., et al., 2010. Microglial activation and increased microglial density observed in the dorsolateral prefrontal cortex in autism. Biol. Psychiatry 68, 368-376.

Moy, S.S., et al., 2007. Mouse behavioral tasks relevant to autism: phenotypes of 10 inbred strains. Behav. Brain Res. 176, 4-20.

Mozhui, K., et al., 2010. Strain differences in stress responsivity are associated with divergent amygdala gene expression and glutamate-mediated neuronal excitability. J. Neurosci. 30, 5357-5367.

Murray, P.J., et al., 2014. Macrophage activation and polarization: nomenclature and experimental guidelines. Immunity $41,14-20$

Nalecz, K.A., et al., 2004. Carnitine: transport and physiological functions in the brain. Mol. Aspects Med. 25, 551-567.

Neefjes, J., et al., 2011. Towards a systems understanding of MHC class I and MHC class II antigen presentation. Nat. Rev. Immunol. 11, 823-836.

Nelson, L.H., Lenz, K.M., 2017. Microglia depletion in early life programs persistent changes in social, mood-related, and locomotor behavior in male and female rats. Behav. Brain Res. 316, 279-293.

Neumann, H., et al., 1998. Neurotrophins inhibit major histocompatibility class II inducibility of microglia: involvement of the p75 neurotrophin receptor. Proc. Natl. Acad. Sci. U. S. A. 95, 5779-5784

Niraula, A., Sheridan, J.F., Godbout, J.P., 2017. Microglia priming with aging and stress. Neuropsychopharmacology 42, 318-333.

Norton, S., et al., 2014. Potential for primary prevention of Alzheimer's disease: an analysis of population-based data. Lancet Neurol. 13, 788-794.

Nunes, S.O., et al., 2013. The shared role of oxidative stress and inflammation in major depressive disorder and nicotine dependence. Neurosci. Biobehav. Rev. 37, 1336-1345.

Offen, D., et al., 1996. Prevention of dopamine-induced cell death by thiol antioxidants: possible implications for treatment of Parkinson's disease. Exp. Neurol. 141, 32-39.

Onasanwo, S.A., et al., 2016. Inhibition of neuroinflammation in BV2 microglia by the biflavonoid kolaviron is dependent on the Nrf2/ARE antioxidant protective mechanism. Mol. Cell. Biochem. 414, 23-36.

Orihuela, R., McPherson, C.A., Harry, G.J., 2016. Microglial M1/M2 polarization and metabolic states. Br. J. Pharmacol. 173, 649-665.

Oxenkrug, G.F., 2010. Tryptophan kynurenine metabolism as a common mediator of genetic and environmental impacts in major depressive disorder: the serotonin hypothesis revisited 40 years later. Isr. J. Psychiatry Relat. Sci. 47, 56-63.

Ozcan, M.E., et al., 2004. Antioxidant enzyme activities and oxidative stress in affective disorders. Int. Clin. Psychopharmacol. 19, 89-95.

Pacelli, C., et al., 2015. Elevated mitochondrial bioenergetics and axonal arborization size are key contributors to the vulnerability of dopamine neurons. Curr. Biol. 25, 2349-2360.

Paolicelli, R.C., et al., 2011. Synaptic pruning by microglia is necessary for normal brain development. Science 333, 1456-1458.

Paolicelli, R.C., Bisht, K., Tremblay, M.E., 2014. Fractalkine regulation of microglial physiology and consequences on the brain and behavior. Front. Cell. Neurosci. 8, 129

Parkhurst, C.N., et al., 2013. Microglia promote learning-dependent synapse formation through brain-derived neurotrophic factor. Cell 155, 1596-1609.

Pearson-Leary, J., Osborne, D.M., McNay, E.C., 2015. Role of glia in stress-induced enhancement and impairment of memory. Front. Integr. Neurosci. 9, 63.

Pettegrew, J.W., Levine, J., McClure, R.J., 2000. Acetyl-L-carnitine physical-chemical, metabolic, and therapeutic properties: relevance for its mode of action in Alzheimer's disease and geriatric depression. Mol. Psychiatry 5, 616-632.

Pfau, M.L., Russo, S.J., 2015. Peripheral and central mechanisms of stress resilience. Neurobiol. Stress 1, 66-79.

Pfeiffer, T., Avignone, E., Nägerl, U.V., 2016. Induction of hippocampal long-term potentiation increases the morphological dynamics of microglial processes and prolongs their contacts with dendritic spines. Sci. Rep. 6, 32422.

Polidori, M.C., Nelles, G., 2014. Antioxidant clinical trials in mild cognitive impairment and Alzheimer's disease - challenges and perspectives. Curr. Pharm. Des. 20, 3083-3092.

Prenderville, J.A., et al., 2015. Adding fuel to the fire: the impact of stress on the ageing brain. Trends Neurosci. 38, 13-25. 
Prinz, M., Priller, J., 2014. Microglia and brain macrophages in the molecular age: from origin to neuropsychiatric disease. Nat. Rev. Neurosci. 15, 300-312.

Ramirez, K., et al., 2015. Imipramine attenuates neuroinflammatory signaling and reverses stress-induced social avoidance. Brain Behav. Immun. 46, 212-220.

Ramirez, K., Niraula, A., Sheridan, J.F., 2016. GABAergic modulation with classical benzodiazepines prevent stress-induced neuro-immune dysregulation and behavioral alterations. Brain Behav. Immun. 51, 154-168.

Ranjekar, P.K., et al., 2003. Decreased antioxidant enzymes and membrane essential polyunsaturated fatty acids in schizophrenic and bipolar mood disorder patients. Psychiatry Res. 121, 109-122.

Ransohoff, R.M., 2016a. A polarizing question: do M1 and M2 microglia exist? Nat. Neurosci. 19, 987-991.

Ransohoff, R.M., 2016b. How neuroinflammation contributes to neurodegeneration. Science $353,777-783$.

Ransohoff, R.M., El Khoury, J., 2015. Microglia in health and disease. Cold Spring Harb. Perspect. Biol. 8.

Reader, B.F., et al., 2015. Peripheral and central effects of repeated social defeat stress: monocyte trafficking, microglial activation, and anxiety. Neuroscience 289, 429-442.

Reddy, R., Reddy, R., 2011. Antioxidant therapeutics for schizophrenia. Antioxid. Redox Signal. 15, 2047-2055.

Reinke, E., Fabry, Z., 2006. Breaking or making immunological privilege in the central nervous system: the regulation of immunity by neuropeptides. Immunol. Lett. 104 102-109.

Reul, J.M., de Kloet, E.R., 1985. Two receptor systems for corticosterone in rat brain: microdistribution and differential occupation. Endocrinology 117, 2505-2511.

Ribas, G.S., Vargas, C.R., Wajner, M., 2014. L-Carnitine supplementation as a potential antioxidant therapy for inherited neurometabolic disorders. Gene 533 , 469-476.

Ribeiro Xavier, A.L., et al., 2015. A distinct population of microglia supports adult neurogenesis in the subventricular zone. J. Neurosci. 35, 11848-11861.

Ribeiro, B.M., et al., 2013. Evidences for a progressive microglial activation and increase in iNOS expression in rats submitted to a neurodevelopmental model of schizophrenia: reversal by clozapine. Schizophr. Res. 151, 12-19.

Rivas-Arancibia, S., et al., 2015. Oxidative stress-dependent changes in immune responses and cell death in the substantia nigra after ozone exposure in rat. Front. Aging Neurosci. 7, 65 .

Rodrigues, R., Petersen, R.B., Perry, G., 2014. Parallels between major depressive disorder and Alzheimer's disease: role of oxidative stress and genetic vulnerability. Cell. Mol. Neurobiol. 34, 925-949.

Rogers, J.T., et al., 2011. CX3CR1 deficiency leads to impairment of hippocampal cognitive function and synaptic plasticity. J. Neurosci. 31, 16241-16250.

Rojo, A.I., et al., 2014. Redox control of microglial function: molecular mechanisms and functional significance. Antioxid. Redox Signal. 21, 1766-1801.

Salemme, A., et al., 2016. Anti-inflammatory effects and antioxidant activity of dihydroasparagusic acid in lipopolysaccharide-activated microglial cells. Brain Res. Bull. 120, 151-158.

Salter, M.W., Beggs, S., 2014. Sublime microglia: expanding roles for the guardians of the CNS. Cell 158, 15-24.

Sapolsky, R.M., Krey, L.C., McEwen, B.S., 1985. Prolonged glucocorticoid exposure reduces hippocampal neuron number: implications for aging. J. Neurosci. 5, 1222-1227.

Savage, J.C., et al., 2015. Nuclear receptors license phagocytosis by trem $2+$ myeloid cells in mouse models of Alzheimer's disease. J. Neurosci. 35, 6532-6543.

Schafer, D.P., et al., 2012. Microglia sculpt postnatal neural circuits in an activity and complement-dependent manner. Neuron 74, 691-705.

Schiavone, S., et al., 2009. Involvement of NOX2 in the development of behavioral and pathologic alterations in isolated rats. Biol. Psychiatry 66, 384-392.

Schiavone, S., et al., 2012. NADPH oxidase elevations in pyramidal neurons drive psychosocial stress-induced neuropathology. Transl. Psychiatry 2, e111.

Schiavone, S., et al., 2013. Severe life stress and oxidative stress in the brain: from animal models to human pathology. Antioxid. Redox Signal. 18, 1475-1490.

Schnieder, T.P., et al., 2014. Microglia of prefrontal white matter in suicide. J. Neuropathol. Exp. Neurol. 73, 880-890

Sekar, A., et al., 2016. Schizophrenia risk from complex variation of complement component 4. Nature 530, 177-183.

Seo, J.S., et al., 2012. NADPH oxidase mediates depressive behavior induced by chronic stress in mice. J. Neurosci. 32, 9690-9699.

Setiawan, E., et al., 2015. Role of translocator protein density, a marker of neuroinflammation, in the brain during major depressive episodes. JAMA Psychiat. 72, 268-275.

Shaftel, S.S., et al., 2007. Sustained hippocampal IL-1 beta overexpression mediates chronic neuroinflammation and ameliorates Alzheimer plaque pathology. J. Clin. Invest. $117,1595-1604$.

Sheridan, G.K., Murphy, K.J., 2013. Neuron-glia crosstalk in health and disease: fractalkine and CX3CR1 take centre stage. Open Biol. 3,130181.

Shi, Q., et al., 2015. Complement C3-deficient mice fail to display age-related hippocampal decline. J. Neurosci. 35, 13029-13042.

Sica, A., Mantovani, A., 2012. Macrophage plasticity and polarization: in vivo veritas. J. Clin. Invest. 122, 787-795.

Sierra, A., et al., 2010. Microglia shape adult hippocampal neurogenesis through apoptosis-coupled phagocytosis. Cell Stem Cell 7, 483-495.

Sierra, A., et al., 2013. Janus-faced microglia: beneficial and detrimental consequences of microglial phagocytosis. Front. Cell. Neurosci. 7, 6

Sierra, A., et al., 2014. Surveillance, phagocytosis, and inflammation: how never-resting microglia influence adult hippocampal neurogenesis. Neural Plast. 2014, 610343.

Sipe, G.O., et al., 2016. Microglial P2Y12 is necessary for synaptic plasticity in mouse visual cortex. Nat. Commun. 7, 10905

Sitta, A., et al., 2011. Evidence that L-carnitine and selenium supplementation reduces oxidative stress in phenylketonuric patients. Cell. Mol. Neurobiol. 31, 429-436.

Slusarczyk, J., et al., 2015. Prenatal stress is a vulnerability factor for altered morphology and biological activity of microglia cells. Front. Cell. Neurosci. 9, 82

Sluzewska, A., et al., 1995. Interleukin-6 serum levels in depressed patients before and after treatment with fluoxetine. Ann. N. Y. Acad. Sci. 762, 474-476.
Sorce, S., et al., 2014. The role of the NADPH oxidase NOX2 in prion pathogenesis. PLoS Pathog. 10, e1004531.

Spires-Jones, T.L., Hyman, B.T., 2014. The intersection of amyloid beta and tau at synapses in Alzheimer's disease. Neuron 82, 756-771.

Squarzoni, P., et al., 2014. Microglia modulate wiring of the embryonic forebrain. Cell Rep. $8,1271-1279$.

Srivareerat, M., et al., 2009. Chronic psychosocial stress exacerbates impairment of cognition and long-term potentiation in beta-amyloid rat model of Alzheimer's disease. Biol. Psychiatry 65, 918-926.

Stein-Behrens, B.A., Lin, W.J., Sapolsky, R.M., 1994. Physiological elevations of glucocorticoids potentiate glutamate accumulation in the hippocampus. J. Neurochem. 63, 596-602.

Steiner, J., et al., 2008. Immunological aspects in the neurobiology of suicide: elevated microglial density in schizophrenia and depression is associated with suicide. J. Psychiatr. Res. 42, 151-157.

Stevens, K.E., et al., 1996. Genetic correlation of inhibitory gating of hippocampal auditory evoked response and alpha-bungarotoxin-binding nicotinic cholinergic receptors in inbred mouse strains. Neuropsychopharmacology 15, 152-162.

Stevens, B., et al., 2007. The classical complement cascade mediates CNS synapse elimination. Cell 131, 1164-1178.

Sugama, S., et al., 2007. Stress induced morphological microglial activation in the rodent brain: involvement of interleukin-18. Neuroscience 146, 1388-1399.

Sugama, S., et al., 2016. Chronic restraint stress triggers dopaminergic and noradrenergic neurodegeneration: possible role of chronic stress in the onset of Parkinson's disease. Brain Behav. Immun. 51, 39-46.

Takahashi, T., et al., 2002. Corticosterone acutely prolonged N-methyl-d-aspartate receptor-mediated $\mathrm{Ca}^{2+}$ elevation in cultured rat hippocampal neurons. J. Neurochem. 83, 1441-1451.

Takahashi, K., Rochford, C.D., Neumann, H., 2005. Clearance of apoptotic neurons without inflammation by microglial triggering receptor expressed on myeloid cells-2. J. Exp. Med. 201, 647-657.

Takatsuru, Y., et al., 2015. Early-life stress increases the motility of microglia in adulthood. J. Physiol. Sci. 65, 187-194.

Takeuchi, H., Kawashima, R., 2016. Neural mechanisms and children's intellectual development: multiple impacts of environmental factors. Neuroscientist 22, 618-631.

Tata, D.A., Marciano, V.A., Anderson, B.J., 2006. Synapse loss from chronically elevated glucocorticoids: relationship to neuropil volume and cell number in hippocampal area CA3. J. Comp. Neurol. 498, 363-374.

Tay, T.L., et al., 2016. Microglia across the lifespan: from origin to function in brain development, plasticity and cognition. J. Physiol.

Tian, L., et al., 2008. Shedded neuronal ICAM-5 suppresses T-cell activation. Blood 111 $3615-3625$.

Torres, L., et al., 2015. Dynamic microglial modulation of spatial learning and social behavior. Brain Behav. Immun.

Torres-Platas, S.G., et al., 2014. Evidence for increased microglial priming and macrophage recruitment in the dorsal anterior cingulate white matter of depressed suicides. Brain Behav. Immun. 42, 50-59.

Townsend, B.E., Johnson, R.W., 2016. Sulforaphane induces Nrf2 target genes and attenuates inflammatory gene expression in microglia from brain of young adult and aged mice. Exp. Gerontol. 73, 42-48.

Tremblay, M.E., Lowery, R.L., Majewska, A.K., 2010. Microglial interactions with synapses are modulated by visual experience. PLoS Biol. 8, e1000527.

Tremblay, M.E., et al., 2012. Effects of aging and sensory loss on glial cells in mouse visual and auditory cortices. Glia 60, 541-558.

Tynan, R.J., et al., 2010. Chronic stress alters the density and morphology of microglia in a subset of stress-responsive brain regions. Brain Behav. Immun. 24, 1058-1068.

Tyrka, A.R., et al., 2010. Childhood maltreatment and telomere shortening: preliminary support for an effect of early stress on cellular aging. Biol. Psychiatry 67, 531-534.

Ueno, M., Yamashita, T., 2014. Bidirectional tuning of microglia in the developing brain: from neurogenesis to neural circuit formation. Curr. Opin. Neurobiol. 27, 8-15.

Ueno, M., et al., 2013. Layer V cortical neurons require microglial support for survival during postnatal development. Nat. Neurosci. 16, 543-551.

Valero, J., Paris, I., Sierra, A., 2016. Lifestyle shapes the dialogue between environment, microglia, and adult neurogenesis. ACS Chem. Nerosci. 7, 442-453.

Vallet, P., et al., 2005. Neuronal expression of the NADPH oxidase NOX4, and its regulation in mouse experimental brain ischemia. Neuroscience 132, 233-238.

Vallieres, L., et al., 2002. Reduced hippocampal neurogenesis in adult transgenic mice with chronic astrocytic production of interleukin-6. J. Neurosci. 22, 486-492.

van Berckel, B.N., et al., 2008. Microglia activation in recent-onset schizophrenia: a quantitative (R)-[11C]PK11195 positron emission tomography study. Biol. Psychiatry 64, $820-822$.

Vivash, L., O'Brien, T.J., 2016. Imaging microglial activation with TSPO PET: lighting up neurologic diseases? J. Nucl. Med. 57, 165-168.

Voikar, V., et al., 2005. Long-term individual housing in C57BL/6J and DBA/2 mice: assessment of behavioral consequences. Genes Brain Behav. 4, 240-252.

Vollert, C., et al., 2011. Exercise prevents sleep deprivation-associated anxiety-like behavior in rats: potential role of oxidative stress mechanisms. Behav. Brain Res. 224 233-240.

von Bernhardi, R., Eugenin-von Bernhardi, L., Eugenin, J., 2015. Microglial cell dysregulation in brain aging and neurodegeneration. Front. Aging Neurosci. 7, 124.

Wahl, S.M., Wen, J., Moutsopoulos, N., 2006. TGF-beta: a mobile purveyor of immune privilege. Immunol. Rev. 213, 213-227.

Wahlsten, D., 2012. The hunt for gene effects pertinent to behavioral traits and psychiatric disorders: from mouse to human. Dev. Psychobiol. 54, 475-492.

Wake, H., et al., 2009. Resting microglia directly monitor the functional state of synapses in vivo and determine the fate of ischemic terminals. J. Neurosci. 29, 3974-3980

Walker, F.R., et al., 2014. Dynamic structural remodelling of microglia in health and disease: a review of the models, the signals and the mechanisms. Brain Behav. Immun. 37, 1-14. 
Walter, J., 2015. The triggering receptor expressed on myeloid cells 2 - a molecular link of neuroinflammation and neurodegenerative diseases. J. Biol. Chem.

Wang, Y., et al., 2012. IL-34 is a tissue-restricted ligand of CSF1R required for the development of Langerhans cells and microglia. Nat. Immunol. 13, 753-760.

Wang, J., et al., 2015. Biochanin A protects dopaminergic neurons against lipopolysaccharide-induced damage and oxidative stress in a rat model of Parkinson's disease. Pharmacol. Biochem. Behav. 138, 96-103.

Wang, Y., et al., 2016. Forsythiaside A exhibits anti-inflammatory effects in LPS-stimulated BV2 microglia cells through activation of $\mathrm{Nrf} 2 / \mathrm{HO}-1$ signaling pathway. Neurochem. Res. 41, 659-665.

Waschek, J.A., 2013. VIP and PACAP: neuropeptide modulators of CNS inflammation, injury, and repair. Br. J. Pharmacol. 169, 512-523.

Wei, R., Jonakait, G.M., 1999. Neurotrophins and the anti-inflammatory agents interleukin-4 (IL-4), IL-10, IL-11 and transforming growth factor-beta1 (TGF-beta1) down-regulate T cell costimulatory molecules B7 and CD40 on cultured rat microglia. J. Neuroimmunol. 95, 8-18.

Wei, L., et al., 2012. Early life stress inhibits expression of a novel innate immune pathway in the developing hippocampus. Neuropsychopharmacology 37, 567-580.

Wohleb, E.S., Delpech, J.C., 2017. Dynamic cross-talk between microglia and periphera monocytes underlies stress-induced neuroinflammation and behavioral consequences. Prog. Neuropsychopharmacol. Biol. Psychiatry 79, 39-47.

Wohleb, E.S., et al., 2011. beta-Adrenergic receptor antagonism prevents anxiety-like behavior and microglial reactivity induced by repeated social defeat. J. Neurosci. 31 6277-6288.

Wohleb, E.S., et al., 2012. Peripheral innate immune challenge exaggerated microglia ac tivation, increased the number of inflammatory CNS macrophages, and prolonged social withdrawal in socially defeated mice. Psychoneuroendocrinology 37, 1491-1505.

Wohleb, E.S., et al., 2014. Re-establishment of anxiety in stress-sensitized mice is caused by monocyte trafficking from the spleen to the brain. Biol. Psychiatry 75, 970-981.

Wright, G.J., et al., 2000. Lymphoid/neuronal cell surface OX2 glycoprotein recognizes a novel receptor on macrophages implicated in the control of their function. Immunity $13,233-242$.
Yang, R.J., et al., 2008. Variation in mouse basolateral amygdala volume is associated with differences in stress reactivity and fear learning. Neuropsychopharmacology 33, 2595-2604

Yirmiya, R., et al., 2001. Effects of antidepressant drugs on the behavioral and physiological responses to lipopolysaccharide (LPS) in rodents. Neuropsychopharmacology 24, 531-544.

Yirmiya, R., Rimmerman, N., Reshef, R., 2015. Depression as a microglial disease. Trends Neurosci. 38, 637-658.

Yoo, K.Y., et al., 2011. Antioxidant enzymes are differently changed in experimental ischemic hippocampal CA1 region following repeated restraint stress. J. Neurol. Sci. 302, $33-42$

Zagorska, A., et al., 2014. Diversification of TAM receptor tyrosine kinase function. Nat. Immunol. 15, 920-928.

Zhan, Y., et al., 2014. Deficient neuron-microglia signaling results in impaired functional brain connectivity and social behavior. Nat. Neurosci. 17, 400-406.

Zhang, X.Y., et al., 2006. Antioxidant enzymes and lipid peroxidation in different forms of schizophrenia treated with typical and atypical antipsychotics. Schizophr. Res. 81, 291-300.

Zhang, S., et al., 2011. CD200-CD200R dysfunction exacerbates microglial activation and dopaminergic neurodegeneration in a rat model of Parkinson's disease. J. Neuroinflammation 8,154

Zhang, H., et al., 2015. SIRP/CD47 signaling in neurological disorders. Brain Res. 1623, $74-80$.

Zhao, Q., et al., 2015. Phenotypic dysregulation of microglial activation in young offspring rats with maternal sleep deprivation-induced cognitive impairment. Sci. Rep. 5, 9513.

Ziv, Y., Schwartz, M., 2008. Orchestrating brain-cell renewal: the role of immune cells in adult neurogenesis in health and disease. Trends Mol. Med. 14, 471-478.

Zugno, A.I., et al., 2014. Omega-3 prevents behavior response and brain oxidative damage in the ketamine model of schizophrenia. Neuroscience 259, 223-231.

Zujovic, V., et al., 2001. In vivo neutralization of endogenous brain fractalkine increases hippocampal TNFalpha and 8-isoprostane production induced by intracerebroventricular injection of LPS. J. Neuroimmunol. 115, 135-143. 